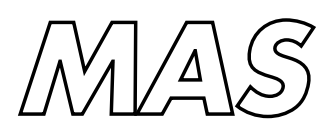

Modelling, Analysis and Simulation

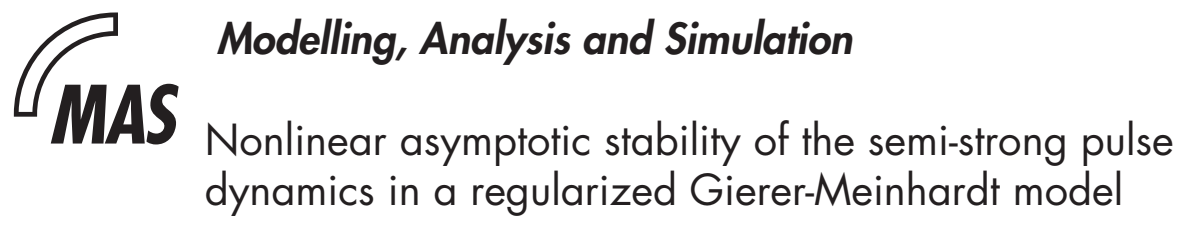

A. Doelman, T.J. Kaper, K. Promislow

Report MAS-E0527 December 2005 
$\mathrm{CWI}$ is the National Research Institute for Mathematics and Computer Science. It is sponsored by the Netherlands Organization for Scientific Research (NWO).

$\mathrm{CWI}$ is a founding member of ERCIM, the European Research Consortium for Informatics and Mathematics.

CWI's research has a theme-oriented structure and is grouped into four clusters. Listed below are the names of the clusters and in parentheses their acronyms.

Probability, Networks and Algorithms (PNA)

Software Engineering (SEN)

\section{Modelling, Analysis and Simulation (MAS)}

Information Systems (INS)

Copyright (C) 2005, Stichting Centrum voor Wiskunde en Informatica

P.O. Box 94079, 1090 GB Amsterdam (NL)

Kruislaan 413, 1098 SJ Amsterdam (NL)

Telephone +31205929333

Telefax +31205924199

ISSN 1386-3703 


\title{
Nonlinear asymptotic stability of the semi-strong pulse dynamics in a regularized Gierer-Meinhardt model
}

\begin{abstract}
We use renormalization group (RG) techniques to prove the nonlinear asymptotic stability for the semi-strong regime of two-pulse interactions in a regularized Gierer-Meinhardt system. In the semi-strong limit the strongly localized activator pulses interact through the weakly localized inhibitor, the interaction is not tail-tail as in the weak interaction limit, and the pulses change amplitude and even stability as their separation distance evolves on algebraically slow time scales. The RG approach employed here validates the interaction laws of quasi-steady pulse patterns obtained formally in the literature, and establishes that the pulse dynamics reduce to a closed system of ordinary differential equations for the activator pulse locations. Moreover, we fully justify the reduction to the nonlocal eigenvalue problem (NLEP) showing the large difference between the quasi-steady NLEP operator and the operator arising from linearization about the pulse is controlled by the resolvent.
\end{abstract}

2000 Mathematics Subject Classification: 35B40, 35K45, 35P15

Keywords and Phrases: Gierer-Meinhardt, semi-strong interaction, renormalization group, geometric singular perturbation, NLEP 



\title{
NONLINEAR ASYMPTOTIC STABILITY OF THE SEMI-STRONG PULSE DYNAMICS IN A REGULARIZED GIERER-MEINHARDT MODEL
}

\author{
ARJEN DOELMAN*, TASSO J. KAPER ${ }^{\dagger}$, AND KEITH PROMISLOW ${ }^{\ddagger}$
}

\begin{abstract}
We use renormalization group (RG) techniques to prove the nonlinear asymptotic stability for the semi-strong regime of two-pulse interactions in a regularized Gierer-Meinhardt system. In the semi-strong limit the strongly localized activator pulses interact through the weakly localized inhibitor, the interaction is not tail-tail as in the weak interaction limit, and the pulses change amplitude and even stability as their spearation distance evolves on algebraically slow time scales. The RG approach employed here validates the interaction laws of quasi-steady pulse patterns obtained formally in the literature, and establishes that the pulse dynamics reduce to a closed system of ordinary differential equations for the activator pulse locations. Moreover, we fully justify the reduction to the nonlocal eigenvalue problem (NLEP) showing the large difference between the quasi-steady NLEP operator and the operator arising from linearization about the pulse is controlled by the resolvent.
\end{abstract}

Key words. Gierer-Meinhardt, semi-strong interaction, renormalization group, geometric singular perturbation, NLEP

AMS subject classifications. 35B40, 35K45, 35P15

1. Introduction. Pulse solutions are the building blocks for the analysis of complex patterns in reaction-diffusion equations. Within the proper scaling limit, the dynamics exhibited by reaction-diffusion systems are governed by the interactions of localized solutions of pulse type. An example is given by the spatio-temporal chaotic dynamics of the one-dimensional Gray-Scott system for which numerical simulations indicate that the chaotic dynamics originate from the interactions and bifurcations of pulse solutions [11].

In the context of singularly perturbed equations in one spatial dimension, there is a well-developed theory of the existence and stability of stationary pulse solutions based on the geometric singular perturbation theory and the Evans function method (see [13,4] and the references therein). There is no such general theory for pulse interactions. In fact, strong pulse interactions, and especially the phenomena of pulse-replication and annihilation, are extensively studied, but not yet understood mathematically. On the other hand, there are methods to study the behavior of pulses in the weak interaction limit where the pulses are so greatly separated that they can be considered at leading order as copies of a solitary pulse. In this regime the exponentially weak interactions only affect the position of the pulses and has no leading order influence on their shape or stability (see $[7,8,12,13]$ and the references therein).

Recently, an intermediate concept has been introduced in the context of singularly perturbed equations, the semi-strong interaction case (see $[5,14]$ and the references therein). The semi-strong regime exists in systems whose components decay at asymptotically distinct rates, so that some of the components of the system approach the trivial background state between pulses, while others are not. Moreover the pulses alter size and shape, see Figure 1.1, and may bifurcate due to the interactions [5, 14]. Up to now the semi-strong pulse interaction has only been studied formally.

In this paper we show that the semi-strong interaction fits naturally into the framework of the renormalization group $(\mathrm{RG})$ methods developed to study the stability of slowly evolving patterns $[12,10]$. For the Gierer-Meinhardt equations, the geometric singular perturbation theory shows that the activator-inhibitor interaction reduces the highly diffusive inhibitor to a local constant within each activator pulse. The value of

\footnotetext{
*Dept. of Modeling, Analysis, and Simulation, CWI, Amsterdam, Netherlands (A.Doelman@cwi.nl).

$\dagger$ Dept. of Mathematics, Boston University, Boston, USA(tasso@math.bu.edu).

${ }^{\ddagger}$ Dept. of Mathematics, Michigan State University, East Lansing, USA (kpromisı@math.msu.edu).
} 
this constant determines the activator pulse interactions. The RG analysis makes these statements rigorous, in particular fully justifying the reductions made in the non-local eigenvalue problem (NLEP) analysis which arises in the linear stability analysis of the pulses.

The singular perturbation theory typically constructs a family of pulse type patterns which are approximate solutions of a given system of equations $[1,2,5]$. The solutions are characterized by parameters $\vec{p} \in \mathbb{R}^{k}$, which are often-but not exclusively-pulse locations. The linearizing about the global manifold of slowly evolving pulse patterns for a particular choice of parameters $\vec{p}$, allows one to decompose the phase space into tangential (or active) and normal (or decaying) modes. In the RG approach, rather than using the exact linearization, reduced linearized operators are identified at a discrete family of base points on the manifold. These form a loose covering of approximate tangent planes, much like the scales of a fish form an piece-wise linear envelope of the underlying body. In the current setting this gives two specific advantages: first, in a neighborhood of each base point we identify a temporally constant linearized operator and associated phase space decomposition, and second we are free to modify the governing linear operator in ways that simplify the analysis. For the Gierer-Meinhardt equations, the singularly perturbed structure of the linearized operators makes them strongly contractive on certain regions of the phase space, this permits a nontrivial replacement of spatially varying potentials with delta functions, affording dramatic simplification to the analysis of the principle linear operator. Indeed we replace the exact linearization with a putatively $\mathcal{O}\left(\varepsilon^{-2}\right)$ "perturbation" which is able to control the flow local to the manifold. These reduced linear operators give rise to exactly the NLEP operators introduced previously in the formal linear stability analysis, $[1,5,14]$.

The RG method shows that the NLEP operators control the flow in a neighborhood of the pulse configurations, generating a thin absorbing set in the phase space. Moreover we recover the leading order pulse evolution by projecting the flow onto the tangent plane of the manifold of two-pulse solutions. In this paper we only consider two-pulse solutions. Although there are no new conceptual features, the generalization to $N$-pulses is technical. In particular determining the amplitudes of each pulse within an $N$-pulse configuration requires a nontrivial nonlinear computation, and the stability of the underlying pattern will depend sensitively upon the pulse shapes and separations. However, the nonlinear aspects of the stability approach we developed here generalizes directly from the two-pulse to the N-pulse case. Our methods can also be applied to semi-strong $N$-pulse interactions in classes of singularly perturbed reaction-diffusion equations as considered in [5]. Nevertheless, interesting additional issues may emerge in specific settings, such as the semi-strong evolution of pulses in the Gray-Scott equation, see [3], in which the essential spectrum is asymtotically close to the origin.

The main result prescribes the semi-strong evolution for the localized two pulses at locations $\boldsymbol{\Gamma}=\left(\Gamma_{1}, \Gamma_{2}\right)^{t}$, capturing as well the impact of transient initial perturbations. After the decay of the transients, we recover the formal pulse velocities at leading order. Fixing $k_{0}>0$ sufficiently large, depending upon $\mu$ but independent of $\varepsilon$, the semi-strong regime is comprised of those pulses whose separations $\Delta=\left|\Gamma_{1}-\Gamma_{2}\right|$ lie in $\left(\Delta \Gamma^{*}(\mu), k_{0} \varepsilon^{-2}\right)$. The lower bound is obtained from the quasi-steady NLEP analysis established in Proposition 3.3, which shows that the two-pulse solution has quasi-stationary eigenvalues which are incompatible with the two-pulse manifold for $\Delta \Gamma<\Delta \Gamma^{*}(\mu)$, this signals the transition to the strong interaction. For the upper bound, the pulse amplitudes $A(\boldsymbol{\Gamma})$, which depend upon the pulse positions $\boldsymbol{\Gamma}$, evolve on an $\mathcal{O}\left(\frac{1}{\varepsilon^{4}}\right)$ time scale, increasing monotonically with growing pulse separation, $\Delta \Gamma$, and approaching the limiting value $\frac{1}{3} \sqrt{\mu}$ for $\Delta \Gamma \gg \varepsilon^{-2}$, see (1.5). We take $k_{0}=k_{0}(\mu)$ so large that the pulse amplitudes and velocities no longer depend upon separation distance, so that $\Delta \Gamma \geq k_{0} \varepsilon^{-2}$ corresponds to the weak pulse interaction regime. In the analysis the upper bound on pulse separation arises from a technical point, see (4.38). An approach which works uniformly for the weak and semi-strong interactions can be obtained by replacing the weighted norm $\|\langle x\rangle \cdot\|_{L^{1}}$ in Lemma 3.1 with a specialized Hölder seminorm on the Fourier transform. However the weak interaction limit has already been studied, see $[7,8,12,13])$, and we avoid this complication, tailoring our analysis to the semi-strong case. 
We introduce the norm $\|\cdot\|_{X}$ on $H^{1} \times H^{1}$ defined by

$$
\|G\|_{X}=\varepsilon\left\|G_{1}\right\|_{L^{2}}+\varepsilon^{-1}\left\|\partial_{\xi} G_{1}\right\|_{L^{2}}+\left\|G_{2}\right\|_{H^{1}},
$$

and remark that it controls the $L^{\infty}$ norm uniformly

$$
\left\|G_{1}\right\|_{L^{\infty}} \leq\left(2\left\|G_{1}\right\|_{L^{2}}\left\|\partial_{\xi} G_{1}\right\|_{L^{2}}\right)^{\frac{1}{2}} \leq \varepsilon\left\|G_{1}\right\|_{L^{2}}+\varepsilon^{-1}\left\|\partial_{\xi} G_{1}\right\|_{L^{2}} \leq\|G\|_{X} .
$$

We state below our main theorem for the pulses in the semi-strong interaction regime.

ThEOREM 1.1. Let $\varepsilon$ be sufficiently small, $\mu>\mu_{\mathrm{Hopf}}$, and the pulse separation $\Delta \Gamma \in\left(\Delta \Gamma^{*}(\mu), k_{0} \varepsilon^{-2}\right)$ where $\Delta^{*}(\mu)$ is as given in Proposition 3.3 and $k_{0}(\mu)>0$ is independent of $\varepsilon$. The manifold of two-pulse solutions (2.5), of the regularized Gierer-Meinhardt equation (2.3) is asymptotically exponentially stable up to $\mathcal{O}\left(\varepsilon^{3}\right)$. That is, given an initial value $\vec{U}_{0}$ sufficiently close to the manifold $\mathcal{M}$ of two-pulse solutions, (3.1), then the corresponding solution $\vec{U}$ of the regularized Gierer-Meinhardt equations satisfies

$$
\vec{U}(\xi, t)=\Phi_{\Gamma}+W(\xi, t),
$$

where $\boldsymbol{\Gamma}(t)$ evolves at leading order according to (4.17) and the remainder $W$ satisfies

$$
\|W\|_{X} \leq M\left(e^{-\nu t}\left\|W_{0}\right\|_{X}+\varepsilon^{3}\right) .
$$

In particular, after the perturbation $W$ has decayed to $\mathcal{O}\left(\varepsilon^{3}\right)$, the pulse evolution is given by the ordinary differential equations (4.70) which are equivalent to

$$
\frac{d}{d t} \Delta \Gamma=\varepsilon^{2} \sqrt{\mu} \frac{e^{-\varepsilon^{2} \Delta \Gamma \sqrt{\mu}}}{1+e^{-\varepsilon^{2} \Delta \Gamma \sqrt{\mu}}},
$$

as obtained formally in [5].

Since the pulses are repelling (1.5), Theorem 1.1 governs the evolution of all asymptotically stable semistrong interacting pulses, i.e. any two-pulse solutions with $\Delta \Gamma(0)>\Delta \Gamma^{*}(\mu)$, will evolve according to (1.5) until they enter the weak intraction regime.

2. The Two-Pulse Solutions of the Gierer-Meinhardt equations. As proposed the GiererMeinhardt model, [9], has an artificial singularity in its nonlinear term, which suggests infinite production of the activator, $V$, in the absence of the inhibitor, $U$. While the singular model can be studied by working with exponentially weighted norms which preserve positivity of the inhibitor, the behavior of the model for small concentrations bears little resemblance to chemical reality. Moreover the singularity has vanishingly small impact on both the two-pulse construction and their evolution. To avoid needless clouding of the analysis we regularize the Gierer-Meinhardt model by truncating the superfluous singularity. In the slow spatial variable $x$, the regularized Gierer-Meinhardt equation is given by,

$$
\left\{\begin{aligned}
U_{t} & =\frac{1}{\varepsilon^{2}} U_{x x}-\mu U+\frac{1}{\varepsilon^{2}} V^{2} \\
V_{t} & =\varepsilon^{2} V_{x x}-V+\frac{V^{2}}{\kappa(U)},
\end{aligned}\right.
$$

where $U(x, t), V(x, t): \mathbb{R} \times \mathbb{R}^{+} \rightarrow \mathbb{R}, \mu>0$ is the main (bifurcation) parameter, and $\varepsilon>0$ is asymptotically small, $0<\varepsilon \ll 1$. The regularizing function $\kappa$ takes the form

$$
\kappa(s)= \begin{cases}s & \text { if } s>2 \delta, \\ \delta & \text { if } 0<s<\delta,\end{cases}
$$

and is smooth for $s \in(\delta, 2 \delta)$, with derivative less than two. In the absence of the inhibitor, $U$, the production rate of the activator $V$ reduces to $V^{2} / \delta$, where $\delta$ is a small parameter. The regularization introduces an 

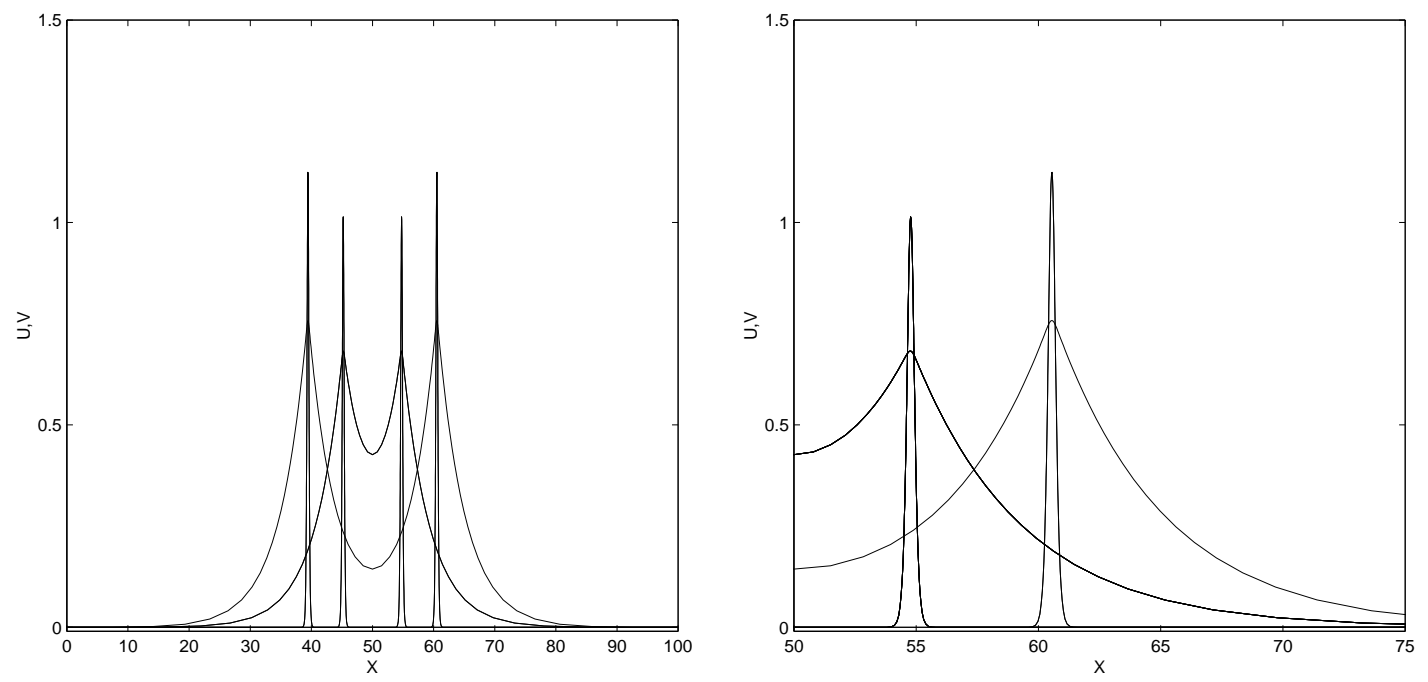

FIG. 1.1. The two-pulse solution of the Gierer-Meinhardt equation, shown at $t=500$ and $t=5000$, in the slow spatial variable. The figures are obtained from numerical simulation (2.1) with $\varepsilon^{2}=0.01$ and $\mu=5$.

$\mathcal{O}\left(e^{-\varepsilon^{-2}|\ln \delta|}\right)$ perturbation to the pulse dynamics. The fast spatial scale is defined by $\xi=\frac{x}{\varepsilon}$, so that (2.1) transforms into

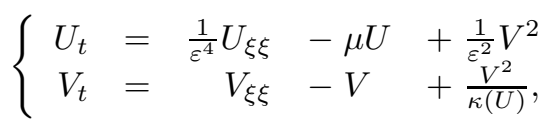

We denote the right-hand side of (2.3) by $F(U, V)$. Since the regularizing term has only an exponentially small impact on the pulse construction we carry over the asymptotic results for the singular Gierer-Meinhardt equation without modification.

Proposition 2.1. The construction and spectral analysis of pulse solutions for the classical GM model given in [4] and the construction and formal dynamics of semi-strong two-pulses given in [5] hold up to exponentially small terms for the regularized models (2.1)/(2.3).

2.1. Notation. We write $f=g+\mathcal{O}(\varepsilon)$ in norm $\|\cdot\|$ if

$$
\|f-g\| \leq c \varepsilon
$$

and assume the $\|\cdot\|_{X}$ norm if no norm is specified. The solution $(U, V)$ of the Gierer-Meinhardt equation is denoted $\vec{U}$. The two-pulse solutions are denoted by $\Phi_{\Gamma}=\left(U_{0}+\varepsilon^{2} U_{2}+\ldots, V_{0}+\varepsilon^{2} V_{2}+\ldots\right)^{t}$, while the initial data of the Gierer-Meinhardt equation is given by $\vec{U}_{0}$. We denote by $\|f\|_{\widehat{L}^{p}}$ and $\|f\|_{\widehat{H}^{2}}$ the $L^{p}$ and $H^{2}$ norms of the Fourier transform of $f$. We remark that $\|f\|_{L^{\infty}} \leq c\|f\|_{\widehat{L}^{1}}$ and conversely $\|f\|_{\widehat{L}^{\infty}} \leq c\|f\|_{L^{1}}$, and in particular that the delta function resides in $\widehat{L}^{\infty}$ but is not in $L^{1}$. Also the norm $\|<x>f\|_{L^{1}}$ with $<x>\equiv 1+|x|$ controls the $L^{\infty}$ norm of the derivative of the fourier transform of $f$. We denote the mass of a function $f$ by $\bar{f}=\int_{-\infty}^{\infty} f d \xi$.

2.2. Asymptotic Pulse Solutions. Within the semi-strong pulse regime the two pulses interact strongly in the inhibitor component, $U$, and weakly through the activator, $V$. To work in this scaling we fix $k_{0}>0$ as large as desired, depending upon $\mu$ but independent of $\varepsilon$. The asymptotic family of semistrong two-pulse solutions is then parameterized by the pulse location $\boldsymbol{\Gamma} \in \mathcal{K}=\left\{\left(\Gamma_{1}, \Gamma_{2}\right) \mid \Gamma_{1}<\Gamma_{2}, k_{0} \varepsilon^{-2} \geq\right.$ 
$\left.\left|\Gamma_{1}-\Gamma_{2}\right| \gg 1 / \sqrt{\varepsilon}\right\}$, where the $1 / \sqrt{\varepsilon}$ bound determines the width of the pulse regions (see below) and the upper bound determines the transition from the semi-strong to the weak pulse interaction. While the lower bound on the pulse separation is large in the fast variables $(\xi)$, it is small, $\mathcal{O}(\sqrt{\varepsilon})$, in the slow $(x)$ variables. The weak pulse interaction, for which $\Delta \Gamma \gg \varepsilon^{-2}$ in the fast variables, has been considered elsewhere. We denote the two-pulse solution by $\Phi_{\Gamma}(\xi)$ which we expand as

$$
\Phi_{\boldsymbol{\Gamma}}(\xi)=\left(\begin{array}{c}
\Phi_{\boldsymbol{\Gamma}, 1} \\
\Phi_{\boldsymbol{\Gamma}, 2}
\end{array}\right)=\left(\begin{array}{c}
U_{0}(\xi ; \boldsymbol{\Gamma})+\varepsilon^{2} U_{2}(\xi ; \boldsymbol{\Gamma})+\varepsilon^{4} U_{4}(\xi ; \boldsymbol{\Gamma}) \\
V_{0}(\xi ; \boldsymbol{\Gamma})+\varepsilon^{2} V_{2}(\xi ; \boldsymbol{\Gamma})
\end{array}\right)
$$

We first describe the leading order terms $\left(U_{0}, V_{0}\right)^{t}$ which were derived in [5]. To fully resolve the pulse dynamics the renormalization procedure of section 4 requires a more accurate description of the two-pulse solution which requires the construction of the higher order corrections, which we outline in Lemma 2.1

In the pulse construction the $V$-components of the two-pulse solutions are centered around the pulsepositions $\xi=\Gamma_{k}(t)$, where

$$
\Gamma_{1}(t)=\Gamma_{0}-\varepsilon^{2} \int_{0}^{t} \hat{c}(s) d s, \quad \Gamma_{2}(t)=\Gamma_{0}+\varepsilon^{2} \int_{0}^{t} \hat{c}(s) d s .
$$

In the two-pulse construction each pulse moves away from their mutual center $\Gamma_{0}$ with equal and opposite speed given by

$$
\hat{c}=\frac{1}{2} \sqrt{\mu} \frac{e^{-\varepsilon^{2} \Delta \Gamma \sqrt{\mu}}}{1+e^{-\varepsilon^{2} \Delta \Gamma \sqrt{\mu}}},
$$

where $\Delta \Gamma=\Delta \Gamma(t)=\left|\Gamma_{1}-\Gamma_{2}\right|$, see (1.5).

The leader order term, $V_{0}$, of the $V$ component of $\Phi_{\Gamma}$ is given by the sum of two one-pulses

$$
V_{0}(\xi ; \Gamma(t))=\phi_{1}+\phi_{2}
$$

where for $k=1,2$ the one pulse solution is

$$
\phi_{k}(\xi)=\frac{3}{2} A(\boldsymbol{\Gamma}) \operatorname{sech}^{2} \frac{1}{2}\left(\xi-\Gamma_{k}(t)\right) .
$$

A key distinction between the semi-strong interaction depicted here and the weak pulse interaction is that the pulse amplitude, $A(\boldsymbol{\Gamma})$, depends nontrivially upon the pulse separation, $\Delta \Gamma=\left|\Gamma_{1}-\Gamma_{2}\right|$, via

$$
A(\boldsymbol{\Gamma})=\frac{\sqrt{\mu}}{3} \frac{1}{1+e^{-2 \varepsilon^{2} \Delta \Gamma \sqrt{\mu}}} .
$$

The pulse regions $\mathcal{I}_{k}=\mathcal{I}_{k}(t), k=1,2$, are defined as regions outside which $V_{0}$ is exponentially small, and such that $U_{0}$ remains constant at leading order over a pulse region. We set the width of the pulse regions to be $\mathcal{O}(1 / \sqrt{\varepsilon})$, i.e. we define

$$
\mathcal{I}_{k}=\left(\Gamma_{k}(t)-\frac{1}{\sqrt{\varepsilon}}, \Gamma_{k}(t)+\frac{1}{\sqrt{\varepsilon}}\right), \quad k=1,2 .
$$

The choice of pulse region width is somewhat arbitrary but standard. Another distinguishing feature of the semi-strong pulse interaction is that the slowly $U$-component of $\Phi_{\Gamma}$ is not the sum of two one-pulses. To the left of $\mathcal{I}_{1}$ and to the right of $\mathcal{I}_{2}, U_{0}(x, t)$ decays super-slowly, while in the region between $\mathcal{I}_{1}$ and $\mathcal{I}_{2}$ it is cosh-like, but again on the slow spatial scale,

$$
U_{0}(\xi ; \boldsymbol{\Gamma})=\left\{\begin{array}{clrl}
A e^{\varepsilon^{2} \sqrt{\mu}\left(\xi-\Gamma_{1}\right)} & \text { for } & \xi & <\Gamma_{1}-\varepsilon^{-\frac{1}{2}} . \\
A \frac{\cosh \varepsilon^{2} \sqrt{\mu}\left(\xi-\left(\Gamma_{1}+\Gamma_{2}\right) / 2\right)}{\cosh \varepsilon^{2} \sqrt{\mu} \Delta \Gamma / 2} & \text { for } \Gamma_{1}+\varepsilon^{-\frac{1}{2}}< & <\Gamma_{2}-\varepsilon^{-\frac{1}{2}}, \\
A e^{-\varepsilon^{2} \sqrt{\mu}\left(\xi-\Gamma_{2}\right)} & \text { for } \Gamma_{2}+\varepsilon^{-\frac{1}{2}}< & \xi
\end{array}\right.
$$


As defined above, $U_{0}$ would be non-smooth if extended into the pulse regions $\mathcal{I}_{k}$. Rather we define the $U$-component of the two-pulse solution inside $\mathcal{I}_{k}$ as $U_{0}+\varepsilon^{2} U_{2}(\xi)$, where $U_{0} \equiv A$, and $U_{2}(\xi)$ is a solution of $U_{\xi \xi}+\phi_{k}^{2}=0-$ see (2.3). Using (2.12) as boundary or matching conditions and the pulse amplitude (2.10), we find

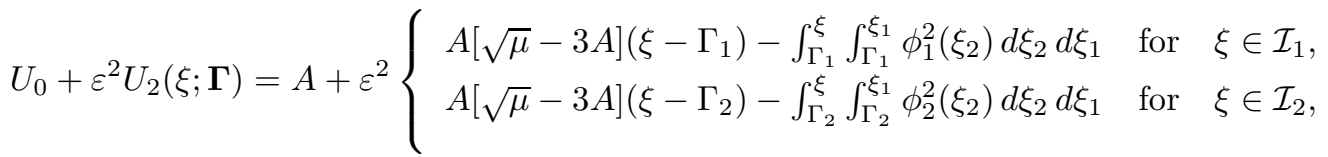

which gives that $U_{0}+\varepsilon^{2} U_{2}(\xi) \in C^{1} \cap H^{2}$. The $C^{1}$-smoothness of $U_{0}+\varepsilon^{2} U_{2}(\xi)$ is equivalent to the amplitudepulse separation relation $(2.10)$, i.e. $U_{0}+\varepsilon^{2} U_{2}(\xi)$ can only be smooth for $A(\boldsymbol{\Gamma})$ given by $(2.10)$.

Relations (2.6), (2.7), (2.8), (2.9), (2.10), (2.12), (2.13) give a leading order description of the two-pulse solution $\Phi_{\boldsymbol{\Gamma}}(\xi)$. The corrections $U_{4}(\xi)$ and $V_{2}(\xi)$ can be obtained by a straightforward regular asymptotic expansion and are both only defined in the pulse regions $\mathcal{I}_{1,2}$ (see (2.22) in the proof of Lemma 2.1 below). The residual of $\Phi_{\Gamma}$,

$$
\mathbf{R}=\mathbf{F}\left(\Phi_{\boldsymbol{\Gamma}}\right)=\left(\begin{array}{c}
F_{1}\left(\Phi_{\boldsymbol{\Gamma}}\right) \\
F_{2}\left(\Phi_{\boldsymbol{\Gamma}}\right)
\end{array}\right)
$$

is determined by the right-hand side of $(2.3)$, denoted by $\left(F_{1}, F_{2}\right)^{t}$, evaluated at $\Phi_{\Gamma}$. Obtaining $L^{1}$ - and $L^{2}$-estimates on the residual is a key step to controlling the remainder in the renormalization process,

LEMMA 2.1. For the residual $\mathbf{R}=\mathbf{F}\left(\Phi_{\boldsymbol{\Gamma}}\right)$ defined in (2.14) we have,

$$
\sup _{\mathbb{R}}\left|F_{2}\left(\Phi_{\boldsymbol{\Gamma}}\right)\right|=\mathcal{O}\left(\varepsilon^{2}\right), \sup _{\mathbb{R} \backslash \mathcal{I}_{1} \cup \mathcal{I}_{2}}\left|F_{1}\left(\Phi_{\boldsymbol{\Gamma}}\right)\right|=\mathcal{O}\left(\varepsilon^{4}\right), \sup _{\mathcal{I}_{1} \cup \mathcal{I}_{2}}\left|F_{1}\left(\Phi_{\boldsymbol{\Gamma}}\right)\right|=\mathcal{O}(\varepsilon \sqrt{\varepsilon}) .
$$

More specifically

$$
R_{2}(\boldsymbol{\Gamma})=\varepsilon^{2} \hat{c}\left(\phi_{1}^{\prime}-\phi_{2}^{\prime}\right)+\mathcal{O}\left(\varepsilon^{4}\right) \text { in } L^{2}(\mathbb{R})
$$

while,

$$
\left\|R_{1}(\boldsymbol{\Gamma})\right\|_{L^{1}}=\mathcal{O}(\varepsilon)
$$

The $\mathcal{O}(\varepsilon \sqrt{\varepsilon})$ bound on $F_{1}$ in $(2.15)$ and the $\mathcal{O}(\varepsilon)$ bound on $R_{1}$ in $(2.17)$ deteriorate to $\mathcal{O}\left(\varepsilon^{-\frac{1}{2}}\right)$ bounds, if we do not introduce the leading order corrections $\varepsilon^{4} U_{4}$ and $\varepsilon^{2} V_{2}$ in (2.5). Moreover, (2.16) no longer holds in that case. On the other hand, the bounds on $R_{2}(\boldsymbol{\Gamma})$ given in the Lemma are sharp. The bounds on $R_{1}(\boldsymbol{\Gamma})$ may be sharpened, but this does not lead to any improvements in the renormalization analysis of section 4 .

Proof: In [5], $\Phi_{\Gamma}$ is constructed as the solution of the classical Gierer-Meinhardt system

$$
\left\{\begin{array}{rlll}
-\varepsilon^{6} \hat{c}_{k} U_{\xi} & =U_{\xi \xi}-\varepsilon^{4} \mu U & +\varepsilon^{2} V^{2} \\
-\varepsilon^{2} \hat{c}_{k} V_{\xi} & =V_{\xi \xi}-V & +\frac{V^{2}}{U}
\end{array}\right.
$$

with $\hat{c}_{1}=-\hat{c}<0$ for $\xi<\Gamma_{0}$ and $\hat{c}_{2}=\hat{c}>0$ for $\xi<\Gamma_{0}$, and $\hat{c}=\hat{c}(t)$ as in (2.7). Note the factor $\varepsilon^{4}$ difference between the right hand sides of the $U$-equation here and in (2.3). We may employ a regular perturbation expansion, writing

$$
\begin{aligned}
& U(\xi, t)=U_{0}(\xi ; \boldsymbol{\Gamma})+\varepsilon^{2} U_{2}(\xi ; \boldsymbol{\Gamma})+\varepsilon^{4} U_{4}(\xi ; \boldsymbol{\Gamma})+\varepsilon^{6} U_{r}\left(\xi, t ; \varepsilon^{2}\right), \\
& V(\xi, t)=V_{0}(\xi ; \boldsymbol{\Gamma})+\varepsilon^{2} V_{2}(\xi ; \boldsymbol{\Gamma})+\varepsilon^{4} V_{r}\left(\xi, t ; \varepsilon^{2}\right),
\end{aligned}
$$

where $A, U_{2}$ and $V_{0}$ are given in (2.8), (2.9), (2.10), (2.12), (2.13), and $U_{4}$ and $V_{2}$ have already been introduced in (2.5). Likewise, we expand $F_{1}$ and $F_{2}$ (Proposition 2.1),

$$
\begin{aligned}
& F_{1}\left(\Phi_{\boldsymbol{\Gamma}}\right)=\frac{1}{\varepsilon^{2}}\left[U_{2, \xi \xi}+V_{0}^{2}\right]+\left[U_{4, \xi \xi}-\mu U_{0}+2 V_{0} V_{2}\right]+\varepsilon^{2}\left[U_{r, \xi \xi}-\varepsilon^{4} \mu U_{r}-F_{1, r}^{\mathrm{inh}}\left(U_{2}, V_{0,2, r} ; \varepsilon^{2}\right)\right] \\
& F_{2}\left(\Phi_{\Gamma}\right)=\left[V_{0, \xi \xi}-V_{0}+\frac{V_{0}^{2}}{U_{0}}\right]+\varepsilon^{2}\left[L_{22} V_{2}-\frac{V_{0}^{2} U_{2}}{U_{0}^{2}}\right]+\varepsilon^{4}\left[L_{22} V_{r}-F_{2, r}^{\mathrm{inh}}\left(U_{0,2,4}, \varepsilon^{2} U_{r}, V_{0,2}, \varepsilon^{2} V_{r} ; \varepsilon^{2}\right)\right]
\end{aligned}
$$


where

$$
L_{22} V=V_{\xi \xi}-V+\frac{V_{0}^{2}}{U_{0}} V
$$

and the expressions for $F_{1, r}^{\mathrm{inh}}\left(U_{2}, V_{0,2, r} ; \varepsilon^{2}\right)$ and $F_{2, r}^{\mathrm{inh}}\left(U_{0,2,4}, \varepsilon^{2} U_{r}, V_{0,2}, \varepsilon^{2} V_{r} ; \varepsilon^{2}\right)$ follow directly by substitution of (2.19) in (2.3). We obtain by (2.18) the following equations for $U_{4}$ and $V_{2}$,

$$
U_{4, \xi \xi}=\mu U_{0}-2 V_{0} V_{2}, \quad L_{22} V_{2}=\frac{V_{0}^{2} U_{2}}{U_{0}^{2}}-\hat{c}_{k} V_{0, \xi}
$$

for $\xi \in \mathcal{I}_{1,2}$. These equations can be solved uniquely by application of the natural boundary/matching conditions. Note that $U_{4}(\xi)$ grows as $\left(\xi-\Gamma_{1,2}\right)^{2}$ for $\left|\xi-\Gamma_{1,2}\right| \gg 1$ and that $V_{2}$ decays exponentially to 0 as $\left|\xi-\Gamma_{1,2}\right| \gg 1$ (see $\left.(2.8)\right)$. The equations for the remainders $U_{r}\left(\xi, t ; \varepsilon^{2}\right)$ and $V_{r}\left(\xi, t ; \varepsilon^{2}\right)$ are given by,

$$
\begin{aligned}
& U_{r, \xi \xi}-\varepsilon^{4} \mu U_{r}=F_{1, r}^{\mathrm{inh}}\left(U_{2}, V_{0,2, r} ; \varepsilon^{2}\right)-\hat{c}_{k} U_{0, \xi}-\varepsilon^{2} \hat{c}_{k} U_{2, \xi}-\varepsilon^{4} \hat{c}_{k} U_{4, \xi}-\varepsilon^{6} \hat{c}_{k} U_{r, \xi}, \\
& L_{22} V_{r} \\
& =F_{2, r}^{\text {inh }}\left(U_{0,2,4}, \varepsilon^{2} U_{r}, V_{0,2}, \varepsilon^{2} V_{r} ; \varepsilon^{2}\right)-\hat{c}_{k} V_{2, \xi}-\varepsilon^{2} \hat{c}_{k} V_{r, \xi}
\end{aligned}
$$

for $\xi \in \mathbb{R}$. It is a straightforward procedure to check that $\left|V_{r}\right|$ and $\left|F_{2, r}^{\text {inh }}\right|$ are uniformly bounded for $\xi \in \mathbb{R}$; in fact, both $V_{r}$ and $F_{2, r}^{\text {inh }}$ decay exponentially to 0 as $\left|\xi-\Gamma_{1,2}(t)\right| \gg 1$. Together with the definitions of $V_{0}$ and $V_{2}((2.8)$ and $(2.22))$, substitution of this result in the second equation of (2.20) yields (2.16). This also implies the results on $F_{2}\left(\Phi_{\boldsymbol{\Gamma}}\right)$ in $(2.15)$.

Outside the pulse regions $\mathcal{I}_{k}$, all $V_{0,2, r}$ components are exponentially small, and $U_{0}$ has been constructed as solution of $U_{\xi \xi}-\varepsilon^{4} \mu U=0$ (2.12). Therefore, the correction $U_{r}$ to $U_{0}$ in the $U$-component of the two-pulse solution also varies as function of $\varepsilon^{2} \xi$, and $U_{2}$ and $U_{4}$ have to be taken $\equiv 0$ outside $\mathcal{I}_{k}$. This implies by (2.20) and (2.23) that outside $\mathcal{I}_{k}$

$$
F_{1}\left(\Phi_{\Gamma}\right)=-\varepsilon^{2} \hat{c}_{k} U_{0, \xi}=\mathcal{O}\left(\varepsilon^{4}\right)
$$

(2.12). Since $U_{r}$ decays for $\xi \rightarrow \pm \infty$ with the same slow rate as $U_{0}$, we find

$$
\int_{\mathbb{R} \backslash \mathcal{I}_{1} \cup \mathcal{I}_{2}}\left|F_{1}\left(\Phi_{\Gamma}\right)\right| d \xi=\frac{1}{\varepsilon^{2}} \times \mathcal{O}\left(\varepsilon^{4}\right)=\mathcal{O}\left(\varepsilon^{2}\right) .
$$

Inside $\mathcal{I}_{k}$, we conclude from (2.23) and the fact that $U_{2}$ grows linearly with $\left(\xi-\Gamma_{i}\right)(2.13)$, that $U_{r}$ may grow as $\left(\xi-\Gamma_{i}\right)^{3}$. Nevertheless, both $U_{r, \xi \xi}$ and $F_{1, r}^{\text {inh }}\left(U_{2}, V_{0,2, r} ; \varepsilon^{2}\right)$ only grow linearly in $\left(\xi-\Gamma_{i}\right)$. Since the width of the $\mathcal{I}_{k}$ intervals is $\mathcal{O}(1 / \sqrt{\varepsilon})(2.11)$, we deduce from $(2.20)$ and $(2.23)$ that $\sup _{\mathcal{I}_{1} \cup \mathcal{I}_{2}}\left|F_{1}\left(\Phi_{\Gamma}\right)\right|=\mathcal{O}_{(\varepsilon \sqrt{\varepsilon})}$ (2.15). Hence, by (2.11),

$$
\int_{\mathcal{I}_{k}}\left|F_{1}\left(\Phi_{\Gamma}\right)\right| d \xi=\frac{1}{\sqrt{\varepsilon}} \times \mathcal{O}(\varepsilon \sqrt{\varepsilon})=\mathcal{O}(\varepsilon),
$$

which yields the $L^{1}$-bound (2.17).

3. Linearization and the Reduced Operators. We define the manifold $\mathcal{M} \subset H^{1} \times H^{1}$ of two-pulse solutions by

$$
\mathcal{M}=\left\{\Phi_{\Gamma} \mid \Gamma \in \mathcal{K}\right\}
$$

We decompose solutions in a neighborhood of the manifold as

$$
\left(\begin{array}{l}
U \\
V
\end{array}\right)=\Phi_{\Gamma}+W(\xi, t)
$$


where the remainder $W=\left(W_{1}, W_{2}\right)^{t}$ and $\boldsymbol{\Gamma}$ is taken as a function of time. In terms of the residual introduced in (3.2) the GM equation (2.3) can then be written as

$$
W_{t}+\frac{\partial \Phi}{\partial \boldsymbol{\Gamma}} \dot{\boldsymbol{\Gamma}}=\mathbf{R}+L_{\boldsymbol{\Gamma}} W+\mathcal{N}(W),
$$

where $R$ is the residual (3.34), $L_{\Gamma}$ is the linearization of $F$ about $\Phi_{\Gamma}$, given by

$$
L_{\boldsymbol{\Gamma}}=\left(\begin{array}{cc}
\epsilon^{-4} \partial_{\xi}^{2}-\mu & 2 \varepsilon^{-2} \Phi_{\Gamma, 2} \\
-\frac{\Phi_{\Gamma, 2}^{2} \kappa^{\prime}\left(\Phi_{\Gamma, 1}\right)}{\kappa\left(\Phi_{\Gamma, 1}\right)^{2}} & \partial_{\xi}^{2}-1+2 \frac{\Phi_{\Gamma, 2}}{\kappa\left(\Phi_{\Gamma, 1}\right)}
\end{array}\right) .
$$

In the linear operator above $\kappa\left(\Phi_{\Gamma, 1}\right)=\Phi_{\Gamma, 1}$ except for those $\xi$ for which $\Phi_{\Gamma, 2}(\xi)=\mathcal{O}\left(e^{-\varepsilon^{-2}|\ln \delta|}\right)$, thus the perturbation to the linearization introduced by the regularization is compact and exponentially small. The final term, $\mathcal{N}(W)$, representing the nonlinearity is given at leading order by

$$
\mathcal{N}(W)=\left(\begin{array}{c}
\epsilon^{-2} W_{2}^{2} \\
\mathcal{O}\left(W_{2}^{2}\right)+\mathcal{O}\left(V_{0} W_{1} W_{2}\right)+\mathcal{O}\left(V_{0}^{2} W_{1}^{2}\right)
\end{array}\right) .
$$

From the asymptotic form of the pulse solution given in (2.8), (2.12), and (2.10), we calculate that

$$
\varepsilon^{2}\left\|\frac{\partial U_{0}}{\partial \Gamma_{k}}\right\|_{L^{1}}+\varepsilon\left\|\frac{\partial U_{0}}{\partial \Gamma_{k}}\right\|_{L^{2}}+\left\|\frac{\partial U_{0}}{\partial \Gamma_{k}}\right\|_{L^{\infty}}=\mathcal{O}\left(\varepsilon^{2}\right),
$$

while

$$
\frac{\partial V_{0}}{\partial \Gamma_{k}}=-\phi_{k}^{\prime}+\mathcal{O}\left(\varepsilon^{2}\right)
$$

in $L^{2}$.

3.1. The Reduced Linearization. A key step in the renormalization group treatment is the replacement of the exact linear operator with a reduced operator whose spectral and semi-group properties are easier to analyze, yet such that the difference between the exact and the reduced operator, the secularity, does not lead to growth of the remainder $W$. Due to the contractivity of the $L_{11}$ component of $L_{\Gamma}$, the two-pulse potential which comprises the $L_{12}$ component can be replaced with $\delta$ functions located at each pulse position. The mass of the delta function is chosen to equal the mass of the product of the original potential and the function it operates upon. We also replace the exact two-pulse solution $\Phi_{\Gamma}$ with its leading order approximation $\left(U_{0}, V_{0}\right)^{t}$. With the reductions the linearized operator is given by

$$
\tilde{L}_{\Gamma}=\left(\begin{array}{cc}
\epsilon^{-4} \partial_{\xi}^{2}-\mu & 2 \varepsilon^{-2}\left(\delta_{\Gamma_{1}} \otimes \phi_{1}+\delta_{\Gamma_{2}} \otimes \phi_{2}\right) \\
-\frac{V_{0}^{2}}{A^{2}} & \partial_{\xi}^{2}-1+2 \frac{V_{0}}{A}
\end{array}\right),
$$

where the tensor product of $f_{1}$ and $f_{2}$ is defined by

$$
\left(f_{1} \otimes f_{2}\right) W=\left(f_{2}, W\right)_{L^{2}} f_{1} .
$$

In particular $\delta_{\Gamma_{k}} \otimes \phi_{k}$ represents the tensor product of the $\delta$ function centered at $\xi=\Gamma_{k}$ with $\phi_{k}$. In the analysis below we use the notation

$$
\alpha_{k}(W)=\left(\phi_{k}, W\right)_{L^{2}},
$$

for $k=1,2$. The scalar operators that appear in the upper left entry, respectively lower right, of the matrix $\tilde{L}$ (3.8) will be denoted by $L_{11}$, resp. $L_{22}$, see (2.21). The reduced operator is ostensibly an $\mathcal{O}\left(\varepsilon^{-2}\right)$ perturbation of the original operator. However it is immediately clear that they share the same essential spectrum

$$
\sigma_{\mathrm{ess}}=\{\lambda \in \mathbb{R}: \lambda \leq \max (-1,-\mu)\}
$$


3.2. The Point Spectrum. The two-pulse profiles which comprise the manifold $\mathcal{M}$ are not stationary solutions, and as such it is not self-consistent to determine their linear stability in terms of the spectrum of the associated linearized operator. We say that the two-pulse solution $\Phi_{\boldsymbol{\Gamma}}$ is spectrally compatible with the manifold $\mathcal{M}$ if the spectrum of the associated linear operator can be decomposed into a part contained within the left-half complex plane and a finite-dimensional part whose associated eigenspace approximates the tangent plane of $\mathcal{M}$ at $\boldsymbol{\Gamma}$.

To determine the point spectrum of $\tilde{L}$ we resolve the eigenvalue equation for the inhibitor, $U$, and reduce the eigenvalue problem to a scalar equation for the activator component, $V$, of the eigenfunction. We call this the NLEP equation, see (3.24), and denote the corresponding linear operator by $\mathcal{L}(\lambda, \Delta \Gamma)$. The NLEP operator controls the point spectrum of $\tilde{L}$, to leading order.

Proposition 3.1. Up to multiplicity we have $\sigma_{p}(\tilde{L})=\{\lambda \mid \operatorname{Ker}(\mathcal{L}(\lambda)) \neq 0\}\left(1+\mathcal{O}\left(\varepsilon^{2}\right)\right)$. That is, for each eigenvalue, $\lambda \in \sigma_{p}(\tilde{L})$, with corresponding eigenvector $\Psi=\left(\Psi_{1}, \Psi_{2}\right)^{t}$, there is a $\lambda_{\mathcal{L}}$ and corresponding $\psi$ such that $\mathcal{L}\left(\lambda_{\mathcal{L}}\right) \psi=\lambda_{\mathcal{L}} \psi,\left|\lambda-\lambda_{\mathcal{L}}\right|_{\sim}=\mathcal{O}\left(\varepsilon^{2}\right)$ with $\Psi_{2}=\psi\left(1+\mathcal{O}\left(\varepsilon^{2}\right)\right)$ and $\Psi_{1}$ given by (3.13) up to $\mathcal{O}\left(\varepsilon^{2}\right)$. Moreover the small eigenvalues of $\tilde{L}$ and $\mathcal{L}$ are both exponentially small.

Proof: The eigenvalue problem for the reduced operator is written as

$$
\tilde{L} \Psi=\lambda \Psi,
$$

where $\Psi=\left(\Psi_{1}, \Psi_{2}\right)^{t}$ is a possibly complex two-vector. Since $L_{11}-\lambda$ is invertible for $\lambda \notin(-\infty,-\mu]$, we may solve for $\Psi_{1}$ as

$$
\Psi_{1}=-2 \varepsilon^{-2}\left(\alpha_{1}\left(L_{11}-\lambda\right)^{-1} \delta_{\Gamma_{1}}+\alpha_{2}\left(L_{11}-\lambda\right)^{-1} \delta_{\Gamma_{2}}\right),
$$

where the $\alpha_{k}=\left(\phi_{k}, \Psi_{2}\right)_{L^{2}}$ are as yet undetermined. From the Fourier transform we find

$$
\widehat{\Psi}_{1}(k)=\frac{2}{\sqrt{2 \pi}} \frac{\varepsilon^{2}\left(\alpha_{1} e^{i k \Gamma_{1}}+\alpha_{2} e^{i k \Gamma_{2}}\right)}{k^{2}+\varepsilon^{4}(\mu+\lambda)} .
$$

From the integral relation

$$
\frac{1}{\sqrt{2 \pi}} \int_{-\infty}^{\infty} e^{-i k \xi} \frac{\varepsilon^{2} e^{i k \Gamma}}{k^{2}+\varepsilon^{4}(\mu+\lambda)}=\sqrt{\frac{\pi}{2(\mu+\lambda)}} e^{-\varepsilon^{2} \sqrt{\mu+\lambda|\xi-\Gamma|}},
$$

we may invert the Fourier transform of $\Psi_{1}$ explicitly,

$$
\Psi_{1}(\xi, t)=\alpha_{1} H\left(\lambda, \xi-\Gamma_{1}\right)+\alpha_{2} H\left(\lambda, \xi-\Gamma_{2}\right),
$$

where

$$
H(\lambda, x)=\frac{1}{\sqrt{\mu+\lambda}} e^{-\varepsilon^{2}|x| \sqrt{\mu+\lambda}} .
$$

Eliminating $\Psi_{1}$, the equation for $\Psi_{2}$ reduces to

$$
\left(L_{22}-\lambda\right) \Psi_{2}=\frac{V_{0}^{2}}{A^{2}} \Psi_{1},
$$

see also (2.21). Since $\Psi_{1}$ is a slowly varying function of $\xi$, while each term in $V_{0}$ decays exponentially to zero at an $\mathcal{O}(1)$ rate in $\xi$, we may reduce the equation for $\Psi_{2}$ to

$$
\begin{aligned}
\left(L_{22}-\lambda\right) \Psi_{2} & =\frac{V_{0}^{2}}{A^{2}}\left(\alpha_{1} H\left(\lambda, \xi-\Gamma_{1}\right)+\alpha_{2} H\left(\lambda, \xi-\Gamma_{2}\right)\right), \\
& =\frac{\phi_{1}^{2}+\phi_{2}^{2}}{A^{2}}\left(\alpha_{1} H\left(\lambda, \xi-\Gamma_{1}\right)+\alpha_{2} H\left(\lambda, \xi-\Gamma_{2}\right)\right)+\mathcal{O}\left(e^{-\Delta \Gamma}\right), \\
& =\frac{1}{A^{2} \sqrt{\mu+\lambda}}\left[\phi_{1}^{2}\left(\alpha_{1}+\alpha_{2} E\right)+\phi_{2}^{2}\left(\alpha_{1} E+\alpha_{2}\right)\right]+\mathcal{O}\left(\varepsilon^{2}\right),
\end{aligned}
$$


where

$$
E=E(\Delta \Gamma ; \lambda)=e^{-\varepsilon^{2} \sqrt{\mu+\lambda} \Delta \Gamma} .
$$

In the tensor product notation this is written as

$$
\left(L_{22}-\lambda\right) \Psi_{2}=\frac{1}{A^{2} \sqrt{\mu+\lambda}}\left[\phi_{1}^{2} \otimes\left(\phi_{1}+E \phi_{2}\right)+\phi_{2}^{2} \otimes\left(E \phi_{1}+\phi_{2}\right)\right] \Psi_{2} .
$$

We define the NLEP operator

$$
\mathcal{L}(\lambda, \Delta \Gamma)=L_{22}-\frac{1}{A^{2} \sqrt{\mu+\lambda}}\left[\phi_{1}^{2} \otimes\left(\phi_{1}+E \phi_{2}\right)+\phi_{2}^{2} \otimes\left(E \phi_{1}+\phi_{2}\right)\right] .
$$

This is a compact perturbation of $L_{22}$ and thus is Fredholm, with the same essential spectrum, but is no-longer self adjoint, indeed its adjoint exchanges the roles of the potentials in each tensor product.

Proposition 3.2. Except for the exponentially small eigenvalues, the point spectrum of the NLEP operator $\mathcal{L}$ is given, up to multiplicity, by the zeros of the equation

$$
\mathcal{R}(\lambda)-3 \frac{\sqrt{\mu+\lambda}}{\sqrt{\mu}} \frac{1+e^{-\varepsilon^{2} \sqrt{\mu} \Delta \Gamma}}{1 \pm e^{-\varepsilon^{2} \sqrt{\mu+\lambda} \Delta \Gamma}}=0
$$

where $\mathcal{R}$ is an explicitly known meromorphic function on $\mathbf{C} \backslash(-\infty,-1]$ given by (3.34).

Proof: The spectrum of the NLEP operator $\mathcal{L}$ can be determined explicitly as the zeros of an analytic equation using the methods developed in [4], which we outline below. We introduce $w_{h}(\xi) \geq 0$ as the scaled homoclinic solution of

$$
w_{\xi \xi}-w+w^{2}=0
$$

with its maximum at $\xi=0$. For $k=1,2$ we introduce the translates $w_{h, k}(\xi)=w_{h}\left(\xi-\Gamma_{k}\right)$. Since $\phi_{k}(\xi)=A w_{h, k}(\xi)$ the equations (2.9) and (3.23) can be written as

$$
\frac{d^{2} \Psi_{2}}{d \xi^{2}}-\left[(1+\lambda)-2\left(w_{h, 1}+w_{h, 2}\right)\right] \Psi_{2}=\frac{1}{\sqrt{\mu+\lambda}}\left[w_{h, 1}^{2}\left(\alpha_{1}+\alpha_{2} E\right)+w_{h, 2}^{2}\left(\alpha_{1} E+\alpha_{2}\right)\right],
$$

where $\alpha_{k}=\alpha_{k}\left(\Psi_{2}\right)$ (3.10). Since both the potential of the Schrödinger operator on the left-hand side of the equation, and the inhomogeneous term on the right-hand side consist of disjoint parts localized about $\Gamma_{1}$ and $\Gamma_{2}$, it is natural to decompose $\Psi_{2}$ into

$$
\Psi_{2}=\psi_{1}(\xi)+\psi_{2}(\xi)
$$

where $\psi_{k}$ is localized about $\Gamma_{k}$ and decays exponentially as $\xi$ moves away from $\Gamma_{k}$. The equation (3.27) is equivalent, up to exponentially small terms, to the coupled system,

$$
\left\{\begin{aligned}
\frac{d^{2} \psi_{1}}{d \xi^{2}}-\left[(1+\lambda)-2 w_{h, 1}\right] \psi_{1} & =\frac{w_{h, 1}^{2}}{\sqrt{\mu+\lambda}}\left(\alpha_{1}+\alpha_{2} E\right), \\
\frac{d^{2} \psi_{2}}{d \xi^{2}}-\left[(1+\lambda)-2 w_{h, 2}\right] \psi_{2} & =\frac{w_{h, 2}^{2}}{\sqrt{\mu+\lambda}}\left(\alpha_{1} E+\alpha_{2}\right) .
\end{aligned}\right.
$$

We define $\bar{\psi}=\bar{\psi}(\xi ; \lambda)$ as the uniquely determined bounded solution of

$$
\frac{d^{2} \psi}{d \xi^{2}}-\left[(1+\lambda)-2 w_{h}\right] \psi=w_{h}^{2}
$$


and its translates $\bar{\psi}_{k}(\xi)$ are defined by $\bar{\psi}_{k}(\xi)=\bar{\psi}\left(\xi-\Gamma_{k}\right)$. The functions $\bar{\psi}$ can be determined explicitly, see $[4]$.

We first consider the solution of (3.30) for $\lambda \notin \sigma_{\text {red }}=\left\{\frac{5}{4}, 0,-\frac{3}{4}\right\} \cup(-\infty,-1]$, the spectrum of the operator

$$
L_{\mathrm{red}}=\frac{d^{2}}{d \xi^{2}}-\left(1-2 w_{h}(\xi)\right)
$$

Clearly,

$$
\psi_{1}(\xi)=C_{1} \bar{\psi}_{1}(\xi), \quad \psi_{2}(\xi)=C_{2} \bar{\psi}_{2}(\xi)
$$

for some constants $C_{k}$ that depend on $\lambda$ and $\Delta \Gamma$. Recalling that here $\alpha_{k}=\left(\phi_{k}, \Psi_{2}\right)_{L^{2}}$ and using (3.28), we find

$$
\alpha_{k}=\int_{-\infty}^{\infty} \phi_{k}\left(\psi_{1}+\psi_{2}\right) d \xi=\int_{-\infty}^{\infty} A w_{h, k}\left(C_{1} \bar{\psi}_{1}+C_{2} \bar{\psi}_{2}\right) d \xi=A C_{k} \int_{-\infty}^{\infty} w_{h, k} \bar{\psi}_{k} d \xi=A C_{k} \int_{-\infty}^{\infty} w_{h} \bar{\psi} d \xi
$$

up to asymptotically small corrections. The quantity

$$
\mathcal{R}(\lambda) \equiv \int_{-\infty}^{\infty} w_{h} \bar{\psi} d \xi
$$

is meromorphic for $\lambda \in \mathbb{C} \backslash(-\infty,-1]$, with poles at $\lambda=\frac{5}{4}$ and $\lambda=-\frac{3}{4}$, see [4]. Note that in [4] a more general function, $\mathcal{R}\left(\lambda ; \beta_{1}, \beta_{2}\right)$, has been defined and studied; (3.34) is related to [4] by $\mathcal{R}(\lambda)=216 \mathcal{R}(\lambda ; 2,2)$. System (3.29) can be written as

$$
\left\{\begin{aligned}
\frac{d^{2} \psi_{1}}{d \xi^{2}}-\left[(1+\lambda)-2 w_{h, 1}\right] \psi_{1} & =\frac{A w_{h, 1}^{2} \mathcal{R}}{\sqrt{\mu+\lambda}}\left(C_{1}+C_{2} E\right) \\
\frac{d^{2} \psi_{2}}{d \xi^{2}}-\left[(1+\lambda)-2 w_{h, 2}\right] \psi_{2} & =\frac{A w_{h, 2}^{2} \mathcal{R}}{\sqrt{\mu+\lambda}}\left(C_{1} E+C_{2}\right) .
\end{aligned}\right.
$$

Comparing the equations for $\psi_{1,2}(\xi)$ to $(3.30)$, we obtain the following equations for $C_{1}$ and $C_{2}$,

$$
C_{1}=\frac{A \mathcal{R}}{\sqrt{\mu+\lambda}}\left(C_{1}+C_{2} E\right), \quad C_{2}=\frac{A \mathcal{R}}{\sqrt{\mu+\lambda}}\left(C_{1} E+C_{2}\right)
$$

or, equivalently,

$$
\left(\begin{array}{cc}
\frac{A \mathcal{R}}{\sqrt{\mu+\lambda}}-1 & \frac{A E \mathcal{R}}{\sqrt{\mu+\lambda}} \\
\frac{A E \mathcal{R}}{\sqrt{\mu+\lambda}} & \frac{A \mathcal{R}}{\sqrt{\mu+\lambda}}-1
\end{array}\right)\left(\begin{array}{l}
C_{1} \\
C_{2}
\end{array}\right)=\left(\begin{array}{l}
0 \\
0
\end{array}\right)
$$

For (3.27) to have non-trivial solutions the determinant of the matrix on the left-hand side of (3.37) must be zero. Isolating $\mathcal{R}(\lambda)$ from the resulting expression and using (2.10) and (3.22), we obtain the equation (3.25) whose zeros are the eigenvalues of the NLEP equation (3.23) up to multiplicity, outside of $\sigma_{\text {red }}$. These eigenvalues lie on curves $\lambda(\Delta \Gamma)$, parametrized by the pulse separation.

For $\lambda \in \sigma_{\text {red }}$ we analyze the eigenvalue equation case by case. For $\lambda=-\frac{3}{4}$ or $\frac{5}{4}$, equation (3.30) does not have a bounded solution since the right-hand side is not orthogonal to the kernel of the $L_{\text {red, }}$, and so these values cannot be eigenvalues. However, the eigenfunction $\frac{d}{d \xi} w_{h}$ of $L_{\text {red }}$ at $\lambda=0$, is $L^{2}$ orthogonal to $w_{h}$. The equation (3.33) implies that $\alpha_{1}=\alpha_{2}=0$ and the system (3.29) has a double eigenvalue at $\lambda=0$ with a two-dimensional eigenspace spanned by $\left\{\frac{d}{d \xi} w_{h}\left(\xi-\Gamma_{1}\right), \frac{d}{d \xi} w_{h}\left(\xi-\Gamma_{2}\right)\right\}$. These eigenvalues do not occur as solutions of $(3.25)$, rather they correspond to exponentially small eigenvalues of the original NLEP system 

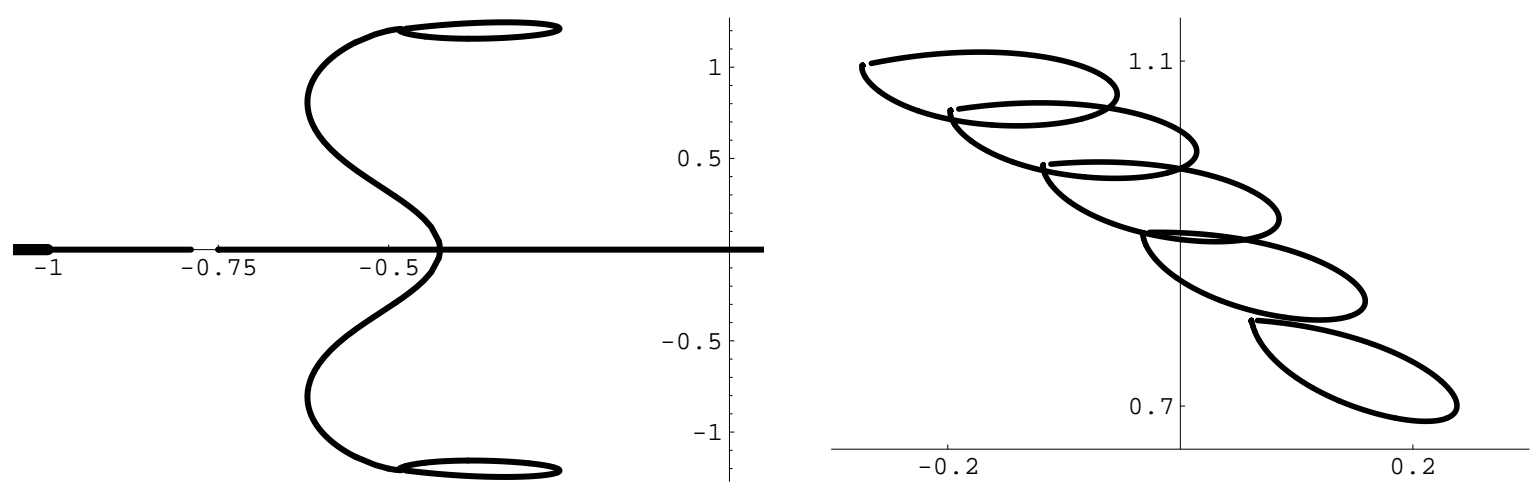

FIG. 3.1. (a) The orbits of the zeroes $\lambda(\Delta \Gamma)$ of (3.25) plotted parametrically in the complex plane as a function of $\Delta \Gamma$ for $\mu=1$. The eigenvalues $\lambda_{+}^{1,2}(\Delta \Gamma)$ are closed loops attached to the homoclinic limit $\lambda \approx-0.48+1.20 i$; the curves $\lambda_{-}^{1,2}(\Delta \Gamma)$ approach the homoclinic point in the limit $\Delta \Gamma \rightarrow \infty$, but $\lambda_{-}^{1,2}(\Delta \Gamma)$ collide on the real axis, becoming real as $\Delta \Gamma$ decreases approaching the limits $\lambda_{-}^{1,2}(\Delta \Gamma) \rightarrow-\frac{3}{4}, \frac{5}{4}$ as $\Delta \Gamma \rightarrow 0$. The third pair of eigenvalues satisfies $\lambda_{ \pm}^{3}(\Delta \Gamma)<-\frac{3}{4}$ with $\lambda_{-}^{3}(\Delta \Gamma)$ disappearing into the essential spectrum of (3.23) as $\Delta \Gamma$ decreases through a critical value. All eigenvalues $\lambda_{ \pm}^{1,2, j}(\Delta \Gamma)$ have negative real part for $\Delta \Gamma>\Delta \Gamma^{*}(1)$ given by (3.38). (b) The closed $\lambda_{+}^{1}(\Delta \Gamma)$-loops for five values of $\mu$ : $\mu=0.7>\mu_{\mathrm{TP}}$, $\mu=0.6,0.5,0.4 \in\left(\mu_{\mathrm{Hopf}}, \mu_{\mathrm{TP}}\right)$ and $\mu=0.3<\mu_{\mathrm{Hopf}}$. The $\Delta \Gamma$-region $\left(\Delta \Gamma_{+}^{1, *}(\mu), \Delta \Gamma_{+}^{2, *}(\mu)\right)$ in which $\operatorname{Re}\left[\lambda_{+}^{1}(\Delta \Gamma)\right]>0$ grows as $\mu \in\left(\mu_{\mathrm{Hopf}}, \mu_{\mathrm{TP}}\right)$ decreases, so that $\Delta \Gamma^{*}(\mu)=\Delta \Gamma_{+}^{2, *}(\mu)$ for $\mu<\mu^{*} \in\left(\mu_{\mathrm{Hopf}}, \mu_{\mathrm{TP}}\right)$.

(3.23), whose corresponding eigenfunctions, derived in Lemma 3.7, form the key spectral projection onto the active tangent plane.

We identify conditions on $\mu$ and $\boldsymbol{\Gamma}$ such that the reduced linearized operator, $\tilde{L}_{\boldsymbol{\Gamma}}$, is spectrally compatible with the manfold $\mathcal{M}$ of two-pulse solutions.

Proposition 3.3. For each $\Delta \Gamma \in(0, \infty)$, the NLEP eigenvalue problem (3.23) has two exponentially small eigenvalues, denoted $\lambda_{ \pm}$, and 4 or 6 eigenvalues $\lambda_{+}^{j_{+}}(\Delta \Gamma)$ and $\lambda_{-}^{j_{-}}(\Delta \Gamma), j_{ \pm}=1, \ldots, J_{ \pm}, J_{ \pm}=J_{ \pm}(\mu)=$ 2 or 3. For all $\mu>\mu_{\mathrm{Hopf}} \approx 0.36$, there is a $\Delta \Gamma^{*}(\mu)$ and a $\nu>0$ such that

$$
\operatorname{Re}\left[\lambda_{ \pm}^{j_{ \pm}}(\Delta \Gamma)\right]<-\nu<0 \text { for all } \Delta \Gamma \geq \Delta \Gamma^{*}(\mu), j_{ \pm}=1, \ldots, J_{ \pm} .
$$

For $\mu>\mu_{\mathrm{TP}} \approx 0.62$ (the tangent point), $\Delta \Gamma^{*}$ takes the exact form

$$
\Delta \Gamma^{*}(\mu)=\frac{1}{\varepsilon^{2} \sqrt{\mu}} \log 3,
$$

while for $\mu \in\left(\mu_{\mathrm{Hopf}}, \mu_{\mathrm{TP}}\right), \Delta \Gamma^{*}(\mu)$ increases with decreasing $\mu$, with $\Delta \Gamma^{*}(\mu) \rightarrow \infty$ as $\mu \downarrow \mu_{\mathrm{Hopf}}$.

Since the two pulses of $\Phi_{\boldsymbol{\Gamma}}(\xi)$ move away from each other $((2.6),(2.7))$, this result implies that the spectrum of the NLEP operator $\mathcal{L}$ remains in the stable half-plane for all $t \geq 0$ if $\Delta \Gamma(0)>\Delta \Gamma^{*}$.

Proof: We can distinguish two limits, $\Delta \Gamma \rightarrow \infty$ and $\Delta \Gamma \downarrow 0$. The first case represents the situation in which the two pulses of $\Phi_{\Gamma}(\xi)$ are so far apart that the two-pulse solution can be considered as two one pulse solutions, i.e. the two-pulse solution is in the weak interaction limit. In this limit, (3.25) reduces to

$$
\mathcal{R}(\lambda)=3 \frac{\sqrt{\mu+\lambda}}{\sqrt{\mu}}
$$

for both $\lambda_{ \pm}(\Delta \Gamma)$. This is the relation that determines the point spectrum of the solitary one-pulse solution of (2.1), independent of the regularization. We conclude by Theorem 5.11 in [4] that (3.39) only has solutions 
with $\operatorname{Re}(\lambda)<0$ for $\mu>\mu_{\text {Hopf }}$ and that (3.23) always has incompatible eigenvalues if $\mu<\mu_{\text {Hopf }} \approx 0.36$. Moreover, (3.39) has 2 or 3 nontrivial eigenvalues, i.e. $\lambda \neq 0$, depending on $\mu$ - the third (compatible) eigenvalue is created in an edge bifurcation as $\mu$ increases through $\mu_{\text {edge }} \approx 0.77$ [4]. There also are 2 or 3 curves $\lambda_{+}^{j_{+}}(\Delta \Gamma)$ and $\lambda_{-}^{j_{-}}(\Delta \Gamma)$, i.e. $j_{ \pm}=1, \ldots, J_{ \pm}, J_{ \pm}(\mu)=2$, respectively 3 , for $\mu<\mu_{\text {edge }}$, resp. $>\mu_{\text {edge }}$. The eigenvalues $\lambda_{ \pm}^{3}(\Delta \Gamma)$ are real and $\lambda_{ \pm}^{3}(\Delta \Gamma)<-\frac{3}{4}$.

For small values of $\Delta \Gamma$ there are two mechanisms to generate incompatible point spectrum, one which occurs for $\mu>\mu_{\mathrm{TP}}$ and the other for $\mu \in\left(\mu_{\mathrm{Hopf}}, \mu_{\mathrm{TP}}\right)$. The first occurs when the eigenvalues $\lambda_{-}^{1}$ and $\lambda_{-}^{2}$ collide and become real. Indeed in the limit $\Delta \Gamma \downarrow 0$, it follows from Proposition 3.2 that $\lambda_{+}^{1,2}(\Delta \Gamma)$ again approaches a solution of (3.39), i.e. the $\lambda_{+}^{1,2}(\Delta \Gamma)$-branches are closed curves. On the other hand, $\left|\mathcal{R}\left(\lambda_{-}^{1,2}(\Delta \Gamma)\right)\right|$ becomes unbounded in this limit. By evaluation of $(3.25)$, we see that $\mathcal{R}(\lambda)$ becomes unbounded as $\lambda_{-}^{1}(\Delta \Gamma) \rightarrow-\frac{3}{4}$, the stable pole of $\mathcal{R}(\lambda)$, and as $\lambda_{-}^{2}(\Delta \Gamma) \rightarrow+\frac{5}{4}$, the other, unstable pole of $\mathcal{R}(\lambda)$. The passage of the real eigenvalue $\lambda_{-}^{2}(\Delta \Gamma)$ through zero corresponds to $\Delta \Gamma$ given by (3.38) since $\mathcal{R}(0)=6$ [4]. In particular, the eigenvalue problem (3.23) has incompatible eigenvalues for all $\Delta \Gamma<\log 3 /\left(\varepsilon^{2} \sqrt{\mu}\right)$, for $\mu>\mu_{\mathrm{TP}}$.

In the second case, the $\lambda_{+}^{1,2}(\Delta \Gamma)$-branches may cross through the imaginary axis. For the tangent point value, $\mu=\mu_{\mathrm{TP}}$, the $\lambda_{+}^{1,2}$ curves are tangent to the imaginary axis. For $\mu \in\left(\mu_{\mathrm{Hopf}}, \mu_{\mathrm{TP}}\right)$, a part of the closed, complex conjugate $\lambda_{+}^{1,2}(\Delta \Gamma)$-curves lies in the unstable half-plane, while the endpoints of the curve, i.e. the eigenvalues associated to the stationary homoclinic one-pulse limit, lies in the stable half-plane, see Figure 3.1(b). More specifically, $\operatorname{Re}\left[\lambda_{+}^{1,2}(\Delta \Gamma)\right]>0$ for $\Delta \Gamma \in\left(\Delta \Gamma_{+}^{1, *}(\mu), \Delta \Gamma_{+}^{2, *}(\mu)\right)$, where

$$
\lim _{\mu \rightarrow \mu_{\mathrm{Hopf}}} \Delta \Gamma_{+}^{1, *}(\mu)=0, \lim _{\mu \rightarrow \mu_{\mathrm{Hopf}}} \Delta \Gamma_{+}^{2, *}(\mu)=\infty, \lim _{\mu \rightarrow \mu_{\mathrm{TP}}} \Delta \Gamma_{+}^{1, *}(\mu)=\lim _{\mu \rightarrow \mu_{\mathrm{TP}}} \Delta \Gamma_{+}^{2, *}(\mu) \approx \frac{1.32}{\varepsilon^{2}},
$$

so that $\Delta \Gamma^{*}(\mu)=\Delta \Gamma_{+}^{2, *}(\mu)>\log 3 /\left(\varepsilon^{2} \sqrt{\mu}\right)$ for $\mu \in\left(\mu_{\mathrm{Hopf}}, \mu_{+}^{*}\right)$ for a certain $\mu_{+}^{*} \in\left(\mu_{\mathrm{Hopf}}, \mu_{\mathrm{TP}}\right)$.

The orbits of the eigenvalues $\lambda$ of (3.23) as function of $\Delta \Gamma$ can be determined by a direct evaluation of $\mathcal{R}(\lambda)$ [4], see Figure 3.1.

REMARK 3.4. Proposition 3.3 implies that $\Phi_{\Gamma}(\xi)$ is not spectrally compatible with the manifold $\mathcal{M}$ if $\Delta \Gamma(0)<\Delta \Gamma^{*}(\mu)$. However this lower bound on the admissible pulse separation distance does not limit the semi-strong character of the pulse interaction in $\Phi_{\Gamma}(\xi)$, since the $U$-component of $\Phi_{\Gamma}(\xi)$ evolves on the slow $\varepsilon^{2} \xi$ space scale. To quantify the lower bound on pulse separation we determine the corresponding maximum value of the minimum $U_{\min }(t)$ of the inhibitor $U$ between the two pulses $\Gamma_{1,2}$ - see also Figure 1.1. Since $U_{\min }(t ; \mu)$ decreases monotonically in time (by $(2.10)$ and $(2.12)$ ), we find that a spectrally compatible two-pulse solution must satisfy

$$
U_{\min }(0)<U_{\min }^{*}(\mu)=\frac{A\left(\Delta \Gamma^{*}\right)}{\cosh \varepsilon^{2} \sqrt{\mu} \Delta \Gamma^{*} / 2}=\frac{3}{16} \sqrt{3 \mu},
$$

if $\mu>\mu_{\mathrm{TP}}$ (3.38). In the context of Figure 1.1, in which $\mu=5$, it follows that $U_{\min }(0)$ must be less than $0.72 \ldots$. The evolution shown there is thus governed by Theorem 1.1.

REMARK 3.5. The lower bound (3.38) on the pulse separation distance does not contradict the pulsesplitting behavior observed in the Gierer-Meinhardt equation [6], in which a stable two-pulse solution is observed with an $\mathcal{O}(1)$ pulse separation distance at the onset of splitting. It is shown in [6] that pulse splitting only occurs for $\mu=\mathcal{O}\left(1 / \varepsilon^{4}\right)$. For these values of $\mu, \Delta \Gamma^{*}(\mu)=\mathcal{O}(1)$ (3.38), which implies that the two $V$-pulses of $\Phi_{\Gamma}(\xi)$ are no longer well-separated. Thus, the lower bound (3.38) agrees with the analysis of [6], since it implies that $\mu$ must be $\mathcal{O}\left(1 / \varepsilon^{4}\right)$ in order to have two-pulse solutions that are not well-separated.

3.3. The Resolvent Estimates and the Semi-group. To establish estimates on the semigroup generated by the reduced linearization $\tilde{L}$ we begin with preliminary bounds on the resolvents of $L_{11}$ and 
$\mathcal{L}$ in the norms defined in Section 2.1. A key point is that the resolvent of $L_{11}$ is strongly contractive on zero-mass functions.

Lemma 3.1. Let $\lambda \in \mathbf{C}$ be an $\mathcal{O}(1)$ distance from $\sigma\left(L_{11}\right)$. Set $g=\left(L_{11}-\lambda\right)^{-1} f$, then the following estimates hold uniformly in $\lambda$,

$$
\varepsilon\|g\|_{L^{2}}+\varepsilon^{-1}\left\|\partial_{\xi} g\right\|_{L^{2}} \leq c \varepsilon^{2}\|f\|_{\widehat{L}^{\infty}} .
$$

Moreover for small total mass, $\bar{f}$ we have the improved estimate,

$$
\varepsilon\|g\|_{L^{2}}+\varepsilon^{-1}\left\|\partial_{\xi} g\right\|_{L^{2}} \leq c\left(\varepsilon^{2}|\bar{f}|+\varepsilon^{4}\|<x>f\|_{L^{1}}\right) .
$$

Proof: We take the Fourier transform of the equation $\left(L_{11}-\lambda\right) g=f$ obtaining,

$$
\widehat{g}(k)=\frac{1}{\sqrt{2 \pi}} \frac{\varepsilon^{4} \widehat{f}(k)}{k^{2}+\varepsilon^{4}(\lambda+\mu)} .
$$

Assuming that $f \in \widehat{L}^{\infty}$, the bound

$$
\left(\int_{-\infty}^{\infty}\left|\frac{\varepsilon^{4}}{k^{2}+\varepsilon^{4}(\lambda+\mu)}\right|^{2} d k\right)^{\frac{1}{2}} \leq c \varepsilon,
$$

for some $c>0$, shows that $\|g\|_{L^{2}} \leq c \varepsilon\|f\|_{\widehat{L}_{\infty}}$. Replacing $\widehat{f}$ with $i k \widehat{f}$ in (3.42) and calculating an integral similar to (3.43) gives $\left\|\partial_{\xi} g\right\|_{L^{2}} \leq c \varepsilon^{3}\|f\|_{\widehat{L}_{\infty}^{\infty}}$. Together these results yield (3.40). In the case that $f$ has small mass, the identify $\widehat{f}(0)=\bar{f}$ and the fact that the norm $\|<x>f\|_{L^{1}}$ controls the $L^{\infty}$ norm of the $k$-derivative of the Fourier transform of $f$, imply that $\widehat{f}$ is uniformly Lipschitz and small at zero, and so we have the estimate

$$
|\widehat{f}(k)| \leq c \frac{|\bar{f}|+|k|}{1+|k|}\|<x>f\|_{L^{1}}
$$

This inequality, used in (3.42) leads to the bound (3.41).

We define $\mathcal{V}$ to be the eigenspace associated to the two exponentially small eigenvalues $\lambda_{ \pm}^{*}$ of $\mathcal{L}^{\dagger}$, the adjoint of $\mathcal{L}$.

Lemma 3.2. Assume that $\lambda \in \mathbf{C}$ is an $\mathcal{O}(1)$ distance from $\sigma(\mathcal{L}) \backslash\left\{\lambda_{+}, \lambda_{-}\right\}$, then we have the following estimate, uniformly in $\lambda$, and for $\Gamma \in \mathcal{K}$.

$$
\left\|(\mathcal{L}-\lambda)^{-1} f\right\|_{H^{1}} \leq c\|f\|_{L^{2}}
$$

for all $f \perp \mathcal{V}$.

Proof: The NLEP operator $\mathcal{L}$ is a finite rank perturbation of $L_{22}$, a self-adjoint Schrödinger operator, and hence is Fredholm. Moreover, away from its point spectrum, $\mathcal{L}-\lambda$ is boundedly invertible with $\mathcal{O}(1)$ inverse, uniformly in $\Delta \Gamma$ for $\Gamma \in \mathcal{K}$. If $f \perp \mathcal{V}$ then $\mathcal{L}-\lambda$ is uniformly invertible for $\lambda$ in a neighborhood of $\lambda_{ \pm}$. To obtain uniformity in $\lambda$ for large $|\lambda|$ we observe that the resolvent of $\mathcal{L}$ can be explicitly constructed in terms of the resolvent of the self-adjoint operator $L_{22}$ and that this later quantity decays like $\left(\operatorname{dist}\left(\lambda, \sigma\left(L_{22}\right)\right)^{-1}\right.$. That the resolvent of $\mathcal{L}$ maps into $H^{1}$ follows from a classic argument by contradiction. 
To study the resolvent of $\tilde{L}$ we project off the eigenspace $\left\{\Psi_{+}, \Psi_{-}\right\}$associated to its small eigenvalues, $\lambda_{ \pm}$. We introduce the space $X_{\Gamma}=\left\{\vec{U} \mid\|\vec{U}\|_{X}<\infty\right.$ and $\left.\pi_{\Gamma} \vec{U}=0\right\}$, where the spectral projection is given in terms of the adjoint eigenfuctions $\Psi_{ \pm}^{\dagger}$ by

$$
\pi_{\Gamma} \vec{U}=\frac{\left(\vec{U}, \Psi_{-}^{\dagger}\right)}{\left(\Psi_{-}, \Psi_{-}^{\dagger}\right)} \Psi_{-}+\frac{\left(\vec{U}, \Psi_{+}^{\dagger}\right)}{\left(\Psi_{+}, \Psi_{+}^{\dagger}\right)} \Psi_{+} .
$$

The complimentary projection is $\tilde{\pi}_{\Gamma}=I-\pi_{\Gamma}$. Assuming the spectral compatibility of $\Phi_{\Gamma}$, the space $X_{\Gamma}$ is associated to temporally decaying solutions of the semigroup generated by $\tilde{L}_{\Gamma}$, while $\tilde{X}_{\Gamma}=\mathcal{R} \pi_{\Gamma}$ is the eigenspace associated to the two exponentially small eigenvalues $\lambda_{ \pm}$. To characterize the projections we need asymptotics for these eigenfunctions.

LEMma 3.3. The small eigenvalue eigenfunctions have the following asymptotic form

$$
\begin{aligned}
& \Psi_{ \pm}=\left(\begin{array}{c}
0 \\
\phi_{1}^{\prime} \pm \phi_{2}^{\prime}
\end{array}\right)+\text { exponentially small } \\
& \Psi_{ \pm}^{\dagger}=\left(\begin{array}{c}
0 \\
\phi_{1}^{\prime} \pm \phi_{2}^{\prime}
\end{array}\right)+\mathcal{O}\left(\varepsilon^{4}\right)
\end{aligned}
$$

in the $X$-norm.

Proof: The expansion for the eigenfunctions follows from classical results. For the adjoint eigenfunctions we present the case for a single pulse, the generalization to two-pulses is straightforward. The adjoint operator is given by

$$
\tilde{L}_{\boldsymbol{\Gamma}}^{\dagger}=\left(\begin{array}{cc}
L_{11} & -\frac{\phi_{1}^{2}}{A^{2}} \\
2 \varepsilon^{-2} \phi_{1} \otimes \delta_{\Gamma_{1}} & L_{22}
\end{array}\right),
$$

where $L_{11}$ and $L_{22}$ are given in (3.8). Writing $\Psi^{\dagger}=\left(\Psi_{1}^{\dagger}, \Psi_{2}^{\dagger}\right)^{t}$, and taking $\lambda_{+}^{*}$ exponentially small, we solve for the second component of $\Psi^{\dagger}$, noting that $\phi_{1}$ is in the range of $L_{22}$ since it is orthogonal to its kernel, $\phi_{1}^{\prime}$,

$$
\Psi_{2}^{\dagger}=\beta \phi_{1}^{\prime}-2 \varepsilon^{-2} \Psi_{1}^{\dagger}\left(\Gamma_{1}\right) L_{22}^{-1} \phi_{1},
$$

where $\beta$ is a free parameter. Solving for $\Psi_{1}^{\dagger}$ we have

$$
\Psi_{1}^{\dagger}=\frac{\beta}{A^{2}} L_{11}^{-1} \phi_{1}^{2} \phi_{1}^{\prime}-\frac{2 \Psi_{1}^{\dagger}\left(\Gamma_{1}\right)}{\varepsilon^{2} A^{2}} L_{11}^{-1}\left(\phi_{1}^{2} L_{22}^{-1} \phi_{1}\right),
$$

The function $\phi_{1}^{2} \phi_{1}^{\prime}$ has zero mass, so from (3.41) we have

$$
\left\|L_{11}^{-1} \phi_{1}^{2} \phi_{1}^{\prime}\right\|_{L^{\infty}} \leq c \varepsilon^{4} .
$$

It can be verified that $\phi_{1}^{2} L_{22}^{-1} \phi_{1}$ is a positive $\mathcal{O}(1)$ function, thus we know $\left.L_{11}^{-1}\left(\phi_{1}^{2} L_{22}^{-1} \phi_{1}\right)\right|_{\xi=\Gamma_{1}}$ is nonzero and $\mathcal{O}(1)$. Evaluating (3.51) at $\xi=\Gamma_{1}$ and solving for $\Psi_{1}^{\dagger}\left(\Gamma_{1}\right)$ shows that $\Psi_{1}^{\dagger}\left(\Gamma_{1}\right)=\mathcal{O}\left(\varepsilon^{6}\right)$. Substituting this back into (3.51) and choosing $\beta=1$ yields the equivalent of (3.48) in the one-pulse case.

With these results we may estimate the resolvent of $\tilde{L}_{\boldsymbol{\Gamma}}$ restricted to $X_{\boldsymbol{\Gamma}}$.

Proposition 3.6. Let $\lambda$ be an $\mathcal{O}(1)$ distance from $\sigma(\tilde{L}) \backslash\left\{\lambda_{+}, \lambda_{-}\right\}$and denote $G=(\tilde{L}-\lambda)^{-1} F$. For $F \in X_{\Gamma}$, we have the following estimates on the resolvent of $\tilde{L}$, holding uniformly in $\lambda$, and in $\Gamma \in \mathcal{K}$,

$$
\|G\|_{X} \leq c\left(\varepsilon^{2}\left\|F_{1}\right\|_{L^{1}}+\left\|F_{2}\right\|_{L^{2}}\right) .
$$


If in addition the mass of $F_{1}$ is small, then we have the improved estimate

$$
\|G\|_{X} \leq c\left(\varepsilon^{2}\left|\bar{F}_{1}\right|+\varepsilon^{4}\left\|<x>F_{1}\right\|_{L^{1}}+\left\|F_{2}\right\|_{L^{2}}\right) .
$$

Proof: By analogy with the eigenvalue problem we solve for $G_{1}$

$$
G_{1}=\left(L_{11}-\lambda\right)^{-1} F_{1}+\alpha_{1} H\left(\lambda, \xi-\Gamma_{1}\right)+\alpha_{2} H\left(\lambda, \xi-\Gamma_{2}\right),
$$

where $H$ is given by (3.17), and $\alpha_{k}=\left(G_{2}, \phi_{k}\right)_{L^{2}}$, for $k=1,2$. The second component of $G$ satisfies

$$
\left(L_{22}-\lambda\right) G_{2}=F_{2}+\frac{V_{0}^{2}}{A^{2}}\left(\left(L_{11}-\lambda\right)^{-1} F_{1}+\alpha_{1} H\left(\xi-\Gamma_{1}\right)+\alpha_{2} H\left(\xi-\Gamma_{2}\right)\right) .
$$

Approximating the product $V_{0}^{2} H$ as in the eigenvalue problem, we find the equation

$$
(\mathcal{L}-\lambda) G_{2}=F_{2}+\frac{V_{0}^{2}}{A^{2}}\left(L_{11}-\lambda\right)^{-1} F_{1},
$$

where the NLEP operator $\mathcal{L}$ is defined in (3.24).

From the asymptotics on $\Psi_{ \pm}^{\dagger}$ the condition $F \in X_{\boldsymbol{\Gamma}}$ is equivalent to the right-hand side of (3.57) being orthogonal to $\mathcal{V}$. From Proposition 3.1 the point spectrum of $\tilde{L}$, less its exponentially small eigenvalues, agrees with the point spectrum of $\mathcal{L}$, less its exponentially small eigenvalues, up to $\mathcal{O}\left(\varepsilon^{2}\right)$. So $\lambda$ is an $\mathcal{O}(1)$ distance from $\sigma(\mathcal{L}) \backslash\left\{\lambda_{ \pm}\right\}$and the estimate (3.45) applied to (3.57) yields

$$
\begin{aligned}
\left\|G_{2}\right\|_{H^{1}} & \leq c\left(\left\|F_{2}\right\|_{L^{2}}+\left\|V_{0}^{2}\left(L_{11}-\lambda\right)^{-1} F_{1}\right\|_{L^{2}}\right), \\
& \leq c\left(\left\|F_{2}\right\|_{L^{2}}+\left\|\left(L_{11}-\lambda\right)^{-1} F_{1}\right\|_{L^{\infty}}\right) .
\end{aligned}
$$

From (3.40) and (1.2) we find that

$$
\left\|G_{2}\right\|_{H^{1}} \leq c\left(\left\|F_{2}\right\|_{L^{2}}+\varepsilon^{2}\left\|F_{1}\right\|_{L^{1}}\right) .
$$

If $F_{1}$ has small mass, then applying (3.41) we have the improved estimates

$$
\left\|G_{2}\right\|_{H^{1}} \leq c\left(\left\|F_{2}\right\|_{L^{2}}+\varepsilon^{2}\left|\bar{F}_{1}\right|+\varepsilon^{4}\left\|<x>F_{1}\right\|_{L^{1}}\right) .
$$

From (3.55) and (3.40) we find that

$$
\left\|G_{1}\right\|_{L^{2}} \leq c\left(\varepsilon\left\|F_{1}\right\|_{L^{1}}+\left\|G_{2}\right\|_{L^{2}}\|H(\lambda)\|_{L^{2}}\right),
$$

but since

$$
|\widehat{H}(k, \lambda)| \leq c\left|\frac{\varepsilon^{2}}{k^{2}+\varepsilon^{4}(\lambda+\mu)}\right|,
$$

we have $\|H(\lambda)\|_{L^{2}} \leq c \varepsilon^{-1}$ and we obtain

$$
\left\|G_{1}\right\|_{L^{2}} \leq c\left(\varepsilon\left\|F_{1}\right\|_{L^{1}}+\varepsilon^{-1}\left\|F_{2}\right\|_{L^{2}}\right) .
$$

A similar argument yields

$$
\left\|\partial_{\xi} G_{1}\right\|_{L^{2}} \leq c\left(\varepsilon^{3}\left\|F_{1}\right\|_{L^{1}}+\varepsilon\left\|F_{2}\right\|_{L^{2}}\right),
$$

which verifies (3.53) 


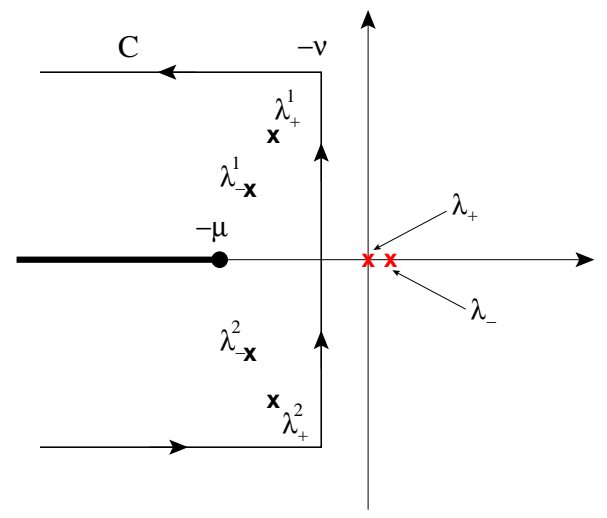

FIG. 3.2. The spectrum, $\sigma(\tilde{L})$, of the reduced operator as determined by Proposition 3.3, and the contour $C$ used to generate the semi-group $S$. Depiction is for the case $\mu>\mu_{\mathrm{Hopf}}$ and $\Delta \Gamma>\Delta \Gamma^{*}(\mu)$ for which $\lambda_{ \pm}^{1,2}$ are within the left-half plane and $J_{ \pm}=2$, see Proposition 3.3. The eigenspace corresponding to the small point spectrum $\left\{\lambda_{ \pm}^{ \pm}\right\}$is projected away and is not contained within the contour.

If $F_{1}$ has small mass, then applying (3.41) to (3.58) yields the improved estimates

$$
\left\|G_{2}\right\|_{H^{1}} \leq c\left(\left\|F_{2}\right\|_{L^{2}}+\varepsilon^{2}\left|\bar{F}_{1}\right|+\varepsilon^{4}\left\|<x>F_{1}\right\|_{L^{1}}\right) .
$$

Following to arguements of (3.61-3.64) yields (3.54).

Since $\tilde{L}$ is an analytic operator we can generate its semigroup from the Laplace transform of the resolvent. We fix the contour $C$ in the complex plane as depicted in Figure 3.3 and

The semigroup $S$ associated to $\left.\tilde{L}\right|_{X_{\Gamma}}$ is given by the contour integral

$$
S(t) F=\frac{1}{2 \pi i} \int_{C} e^{\lambda t}(\lambda-\tilde{L})^{-1} F d \lambda,
$$

where we assume that $F \in X_{\Gamma}$. The semi-group inherits the following properties from the resolvent.

Proposition 3.7. Let $\mu>\mu_{\mathrm{Hopf}}$ and $\Delta \Gamma>\Delta \Gamma^{*}(\mu)$ be given and let $\nu>0$ be as given by Proposition 3.3. The solution $\vec{U}$ of $\vec{U}=S(t) F$, where $F \in X_{\Gamma}$, satisfies

$$
\|\vec{U}\|_{X} \leq M e^{-\nu t}\left(\varepsilon^{2}\left\|F_{1}\right\|_{L^{1}}+\left\|F_{2}\right\|_{L^{2}}\right) .
$$

for some $M>0$ independent of $\Delta \Gamma>\Delta \Gamma^{*}(\mu)$. If in addition $F_{1}$ has small mass, then we have the improved estimate

$$
\|\vec{U}\|_{X} \leq M e^{-\nu t}\left(\varepsilon^{2}\left|\bar{F}_{1}\right|+\varepsilon^{4}\left\|<x>F_{1}\right\|_{L^{1}}+\left\|F_{2}\right\|_{L^{2}}\right) .
$$

Proof: By Proposition 3.3, the conditions on $\mu$ and $\boldsymbol{\Gamma}$ imply that $\sigma(\tilde{L}) \backslash\left\{\lambda_{+}, \lambda_{-}\right\}$is contained within the interior of the contour $C$, and $\operatorname{dist}(\sigma(\tilde{L}), C)=\mathcal{O}(1)$. The estimates on the semigroup follow directly from the contour integral representation (3.66) of $S(t)$, the resolvent estimates (3.53-3.54), and the uniformity of these estimates over the contour $C$. 
4. Nonlinear Stability via the Renormalization Group method. We adapt the renormalization group method developed in [12] to the singular perturbation setting of the Gierer-Meinhardt equations. We assume at time $t_{0}$ that our initial data $\vec{U}_{0}$ satisfies

$$
\left\|\Phi_{\Gamma_{*}}-\vec{U}_{0}\right\| \leq \delta
$$

for some $\boldsymbol{\Gamma}_{*} \in \mathcal{K}$. The following Proposition, adapted from Proposition 2.2 of [12], permits us to choose our base point $\boldsymbol{\Gamma}_{0}$ about which we develop our local coordinate system.

Proposition 4.1. Fix $\delta \ll 1$. Given $\vec{U}_{0}$ and $\boldsymbol{\Gamma}_{*} \in \mathcal{K}$ satisfying $\left\|W_{*}\right\|_{X} \leq \delta$, for $W_{*} \equiv \Phi_{\boldsymbol{\Gamma}_{*}}-\vec{U}_{0}$, then there exists $M>0$, independent of $\vec{U}_{0}$ and $\boldsymbol{\Gamma}_{*}$, and a smooth function $\mathcal{H}: X \mapsto \mathcal{K}$ such that $\boldsymbol{\Gamma}=\boldsymbol{\Gamma}_{*}+\mathcal{H}\left(W_{*}\right)$ satisfies

$$
W_{0} \equiv \vec{U}_{0}-\Phi_{\Gamma} \in X_{\Gamma}
$$

Moreover, if $W_{*} \in X_{\tilde{\boldsymbol{\Gamma}}}$ for some $\tilde{\boldsymbol{\Gamma}} \in \mathcal{K}$ then

$$
\left|\boldsymbol{\Gamma}-\boldsymbol{\Gamma}_{*}\right| \leq M_{0}\left\|W_{*}\right\|_{X}\left|\boldsymbol{\Gamma}_{*}-\tilde{\boldsymbol{\Gamma}}\right| .
$$

Proof: Since

$$
W_{0}=W_{*}+\Phi_{\Gamma}-\Phi_{\Gamma_{*}},
$$

the condition (4.2) is equivalent to

$$
0=\pi_{\Gamma} W_{0}=\pi_{\Gamma}\left(W_{*}+\Phi_{\Gamma}-\Phi_{\Gamma_{*}}\right)
$$

Since $\Psi_{ \pm}^{\dagger}$, are approximately spanned by $\left(0, \phi_{1}^{\prime}\right)^{t}$ and $\left(0, \phi_{2}^{\prime}\right)^{t}$, and $\Phi_{\Gamma, 2}=V_{0}+\mathcal{O}\left(\varepsilon^{2}\right)$, our equations $\Lambda=$ $\left(\Lambda_{1}, \Lambda_{2}\right)^{t}$, are equivalent, up to $\mathcal{O}\left(\varepsilon^{2}\right)$, to

$$
\begin{aligned}
& \Lambda_{1}\left(\boldsymbol{\Gamma}, W_{*}\right) \equiv\left(W_{2, *}+V_{0}(\boldsymbol{\Gamma})-V_{0}\left(\boldsymbol{\Gamma}_{*}\right), \phi_{1}^{\prime}\left(\cdot, \Gamma_{1}\right)\right)_{L^{2}}=0, \\
& \Lambda_{2}\left(\boldsymbol{\Gamma}, W_{*}\right) \equiv\left(W_{2, *}+V_{0}(\boldsymbol{\Gamma})-V_{0}\left(\boldsymbol{\Gamma}_{*}\right), \phi_{2}^{\prime}\left(\cdot, \Gamma_{2}\right)\right)_{L^{2}}=0 .
\end{aligned}
$$

Since $\Lambda\left(\boldsymbol{\Gamma}_{*}, 0\right)=0$ and the $\boldsymbol{\Gamma}$ gradient of $\Lambda$ given by

$$
\left.\nabla_{\boldsymbol{\Gamma}} \Lambda\right|_{\left(\boldsymbol{\Gamma}=\boldsymbol{\Gamma}_{*}, W_{*}=0\right)}=\left(\begin{array}{cc}
-\left\|\phi_{1}^{\prime}\right\|_{L^{2}} & 0 \\
0 & -\left\|\phi_{1}^{\prime}\right\|_{L^{2}}
\end{array}\right)+\mathcal{O}\left(\varepsilon^{2}\right)
$$

is uniformly invertible, the implicit function theorem guarantees the existence of a smooth function $\mathcal{H}$ which provides the solution of (4.2) and in a neighborhood about the manifold $\mathcal{M}$ defined in (3.1). The interval of existence of $\mathcal{H}$ may be chosen uniformly in $\boldsymbol{\Gamma}$ since the solution of (4.2) behaves smoothly as $\Delta \Gamma \rightarrow \infty$.

If in addition we have $W_{*} \in X_{\tilde{\Gamma}}$, then $\left(W_{2, *}, \phi_{k}^{\prime}\left(\tilde{\Gamma}_{k}\right)\right)_{L^{2}}=\mathcal{O}\left(\varepsilon^{4}\right)$ for $k=1,2$. We see that

$$
\left|\left(W_{2, *}, \phi_{k}^{\prime}\left(\Gamma_{k}\right)\right)_{L^{2}}\right| \leq\left|\left(W_{2, *}, \phi_{k}^{\prime}\left(\tilde{\Gamma}_{k}\right)-\phi_{k}^{\prime}\left(\Gamma_{k}\right)\right)_{L^{2}}\right| \leq M_{0}\left\|W_{*}\right\|_{L^{2}}|\tilde{\boldsymbol{\Gamma}}-\boldsymbol{\Gamma}| .
$$


4.1. The Projected Equations. To begin the RG procedure we freeze $\boldsymbol{\Gamma}=\boldsymbol{\Gamma}_{0}$ in $X_{\boldsymbol{\Gamma}_{0}}$, where $\boldsymbol{\Gamma}_{0}$ is the base point provided by the proposition above, and change variables as

$$
\vec{U}(t)=\Phi_{\boldsymbol{\Gamma}}+W
$$

where $W \in X_{\boldsymbol{\Gamma}_{0}}$, and $\boldsymbol{\Gamma}=\boldsymbol{\Gamma}(t)$. Comparing to (3.3), we write the evolution for the remainder $W$ as

$$
\begin{aligned}
W_{t}+\frac{\partial \Phi}{\partial \boldsymbol{\Gamma}} \dot{\boldsymbol{\Gamma}} & =\mathbf{R}+\tilde{L}_{\boldsymbol{\Gamma}_{0}} W+\left(L_{\boldsymbol{\Gamma}}-\tilde{L}_{\boldsymbol{\Gamma}_{0}}\right) W+\mathcal{N}(W), \\
W(\xi, 0) & =W_{0},
\end{aligned}
$$

where $W_{0}=W_{*}+\Phi_{\Gamma_{0}}-\Phi_{\Gamma_{*}}$. The terms $\Delta L \equiv L_{\boldsymbol{\Gamma}}-\tilde{L}_{\boldsymbol{\Gamma}_{0}}$ include both the approximations made to the linear operator and the secular growth implicit in the sliding of $\boldsymbol{\Gamma}$ away from $\boldsymbol{\Gamma}_{0}$.

To enforce $W \in X_{\boldsymbol{\Gamma}_{0}}$ we impose the non-degeneracy condition $\frac{\partial}{\partial t} \pi_{0} W=0$, where $\pi_{0}=\pi_{\boldsymbol{\Gamma}_{0}}$ is given by (3.46). Since $\pi_{0}$ is independent of time, the non-degeneracy condition is equivalent to $\pi_{0} W_{t}=0$, and moreover as $\pi_{0}$ commutes with $\tilde{L}_{\boldsymbol{\Gamma}_{0}}$ it follows that $\pi_{0} \tilde{L}_{\boldsymbol{\Gamma}_{0}} W=\tilde{L}_{\boldsymbol{\Gamma}_{0}} \pi_{0} W=0$. The non-degeneracy condition is thus equivalent to the two equations

$$
\left(\frac{\partial \Phi}{\partial \boldsymbol{\Gamma}} \dot{\boldsymbol{\Gamma}}, \Psi_{ \pm}^{\dagger}\right)_{L^{2}}=\left(\mathbf{R}+\Delta L W+\mathcal{N}(W), \Psi_{ \pm}^{\dagger}\right)_{L^{2}}
$$

From the form of the semi-strong pulse solutions, and assuming momentarily that $\dot{\boldsymbol{\Gamma}}=\mathcal{O}\left(\varepsilon^{2}\right)$, we calculate

$$
\frac{\partial \Phi_{\boldsymbol{\Gamma}}}{\partial \boldsymbol{\Gamma}} \dot{\boldsymbol{\Gamma}}=\left(\begin{array}{c}
0 \\
\phi_{1}^{\prime} \dot{\Gamma}_{1}+\phi_{2}^{\prime} \dot{\Gamma}_{2}
\end{array}\right)+\left(\begin{array}{c}
\mathcal{O}\left(\varepsilon^{3}\right) \\
\mathcal{O}\left(\varepsilon^{4}\right)
\end{array}\right)
$$

component-wise in the $L^{2}$ norm. Using the form of the adjoint eigenvector (3.48) and (3.7), the equations (4.13) may be written

$$
\left(\begin{array}{cc}
\left\|\phi_{1}^{\prime}\right\|_{L^{2}}^{2}+\mathcal{O}\left(\varepsilon^{4}\right) & \left\|\phi_{2}^{\prime}\right\|_{L^{2}}^{2}+\mathcal{O}\left(\varepsilon^{4}\right) \\
\left\|\phi_{1}^{\prime}\right\|_{L^{2}}^{2}+\mathcal{O}\left(\varepsilon^{4}\right) & -\left\|\phi_{2}^{\prime}\right\|_{L^{2}}^{2}+\mathcal{O}\left(\varepsilon^{4}\right)
\end{array}\right) \dot{\boldsymbol{\Gamma}}=\left(\begin{array}{c}
\left(\mathbf{R}+\Delta L W+\mathcal{N}(W), \Psi_{+}^{\dagger}\right. \\
\left(\mathbf{R}+\Delta L W+\mathcal{N}(W), \Psi_{-}^{\dagger}\right)_{L^{2}}
\end{array}\right)
$$

Again using the asymptotic form of the adjoint eigenfunctions $\Psi_{ \pm}^{\dagger}$ we may neglect the contribution from $\Psi_{ \pm, 1}^{\dagger}$ in the inner products on the right-hand side of (4.15). In particular, from the $L^{1}$ bounds on $R_{1}$ from (2.17), we have

$$
\left(R_{1}, \Psi_{ \pm, 1}^{\dagger}\right)_{L^{2}} \leq\left\|R_{1}\right\|_{L^{1}}\left\|\Psi_{ \pm, 1}^{\dagger}\right\|_{L^{\infty}}=\mathcal{O}\left(\varepsilon^{5}\right) .
$$

Inverting the matrix on the left-hand side, and using the expansions for $\Psi_{ \pm, 2}^{\dagger}$ we arrive at the equations of motion for $\boldsymbol{\Gamma}$ which show explicitly the coupling between the remainder $W$ and the pulse evolution,

$$
\dot{\Gamma}_{k}=-\frac{\left(R_{2}+[\Delta L W]_{2}+\mathcal{N}_{2}, \phi_{k}^{\prime}\left(\cdot ; \boldsymbol{\Gamma}_{0}\right)\right)_{L^{2}}}{\left(\phi_{k}^{\prime}, \phi_{k}^{\prime}\right)_{L^{2}}}+\mathcal{O}\left(\varepsilon^{5}, \varepsilon^{4}\|W\|_{X}\right) .
$$

To simplify the equation for the evolution of the remainder, $W$, we introduce the reduced residual

$$
\tilde{\mathbf{R}}=\tilde{\pi}_{\boldsymbol{\Gamma}}\left(\mathbf{R}-\frac{\partial \Phi_{\boldsymbol{\Gamma}}}{\partial \boldsymbol{\Gamma}} \dot{\boldsymbol{\Gamma}}\right)
$$

and observe from the asymptotic description (2.16) of $R_{2}$, that the projection removes the leading order term from the second component of the residual. By Lemma 2.1, the reduced residual enjoys the estimates

$$
\begin{aligned}
\left\|\tilde{R}_{1}\right\|_{L^{1}} & \leq \mathcal{O}(\varepsilon), \\
\left\|\tilde{R}_{2}\right\|_{L^{2}} & \leq \mathcal{O}\left(\varepsilon^{4}\right) .
\end{aligned}
$$


The evolution for the remainder $W$ is now given by

$$
\begin{aligned}
W_{t} & =\tilde{\mathbf{R}}+\tilde{L}_{0} W+\tilde{\pi}_{0}(\Delta L W+\mathcal{N}), \\
W\left(\xi, t_{0}\right) & =W_{0},
\end{aligned}
$$

where $\tilde{L}_{0}=\tilde{L}_{\Gamma_{0}}$ and $\tilde{\pi}_{0}=I-\pi_{\Gamma_{0}}$. The point of the reduction of the Gierer-Meinhardt (2.3) to the projected residual equation (4.21), in the case of two-pulse dynamics, is that the asymptotically relevant and the asymptotically negligible terms are now evident. The evolution for $W$ is controlled by the first two terms on the right-hand side of (4.21), we will show that the last two terms are asymptotically irrelevant, until $\boldsymbol{\Gamma}-\boldsymbol{\Gamma}_{0}$ is so large that the secularity implicit in $\Delta L$ forces an update of $\boldsymbol{\Gamma}_{0}$.

4.2. Decay of the Remainder. We identify the duration of each renormalization interval, and quantify the decay of the remainder $W$ over this interval. To control the dynamics we introduce the quantities

$$
\begin{aligned}
& T_{0}(t)=\sup _{t_{0}<s<t} e^{\nu\left(s-t_{0}\right)}\|W(s)\|_{X}, \\
& T_{1}(t)=\sup _{t_{0}<s<t}\left|\boldsymbol{\Gamma}(s)-\boldsymbol{\Gamma}_{0}\right| .
\end{aligned}
$$

The first enforces the decay of the remainder, $W$, the second measures the distance the pulse positions have moved from its frozen base point. The variation of constants formula applied to (4.21) yields the solution

$$
W(\xi, t)=S(\Delta t) W_{0}+\int_{t_{0}}^{t} S(t-s)\left(\tilde{R}+\tilde{\pi}_{0}(\Delta L W+\mathcal{N})\right) d s,
$$

where we have introduced $\Delta t=t-t_{0}$.

To estimate the distance that the pulse locations, $\boldsymbol{\Gamma}$, have moved from the base point, $\boldsymbol{\Gamma}_{0}$ we examine the equations (4.17). We break $\Delta L$ in secular and reductive parts, $\Delta L=\Delta L_{s}+\Delta L_{r}$ where $\Delta L_{s}=L_{\boldsymbol{\Gamma}}-L_{\Gamma_{0}}$ and $\Delta L_{r}=L_{\Gamma_{0}}-\tilde{L}_{\Gamma_{0}}$, and remark that,

$$
\begin{aligned}
\left\|[\Delta L W]_{2}\right\|_{L^{2}} & \leq\left\|\left[\Delta L_{s} W\right]_{2}\right\|_{L^{2}}+\left\|\left[\Delta L_{r} W\right]_{2}\right\|_{L^{2}}, \\
& \leq c\left(\left|\boldsymbol{\Gamma}-\boldsymbol{\Gamma}_{0}\right|+\varepsilon^{2}\right)\|W\|_{L^{2}}, \\
& \leq c\left(T_{1}(t)+\varepsilon^{2}\right) e^{-\nu\left(t-t_{0}\right)} T_{0}(t),
\end{aligned}
$$

where the estimates on $\Delta L_{s}$ and $\Delta L_{r}$ are described in more detail below. From the form (3.5) of the regularized nonlinearity it is straightforward to obtain the estimate

$$
\left|\left(\mathcal{N}_{2}, \phi_{k}^{\prime}\right)_{L^{2}}\right| \leq c\|W\|_{X}^{2} .
$$

With these bounds in hand, the drift of the pulses is controlled by their speed,

$$
\begin{aligned}
T_{1}(t) \leq \int_{t_{0}}^{t_{0}+\Delta t}|\dot{\boldsymbol{\Gamma}}(s)| d s & \leq \int_{t_{0}}^{t_{0}+\Delta t} c\left(\left\|R_{2}\right\|_{L^{2}}+\left(\varepsilon^{2}+T_{1}(t)\right) e^{-\nu\left(s-t_{0}\right)} T_{0}(t)+e^{-2 \nu\left(s-t_{0}\right)} T_{0}^{2}(t)\right) d s, \\
& \leq c\left(\varepsilon^{2} \Delta t+\left(\varepsilon^{2}+T_{1}\right) T_{0}+T_{0}^{2}\right) .
\end{aligned}
$$

For $T_{0}$ small enough we can eliminate $T_{1}$ from the right-hand side, and neglecting $T_{0}$ in the sum $T_{0}+\Delta t$, we obtain

$$
T_{1} \leq c\left(\varepsilon^{2} \Delta t+T_{0}^{2}\right)
$$



form

Turning to bounds on the remainder, we estimate the irrelevant terms first. The secular term takes the

$$
\Delta L_{s}=\left(\begin{array}{cc}
0 & 2 \varepsilon^{-2}\left(V_{0}(\cdot ; \boldsymbol{\Gamma})-V_{0}\left(\cdot ; \boldsymbol{\Gamma}_{0}\right)\right) \\
V_{12}(\cdot ; \boldsymbol{\Gamma})-V_{12}\left(\cdot, \boldsymbol{\Gamma}_{0}\right) & V_{22}(\cdot ; \boldsymbol{\Gamma})-V_{22}\left(\cdot ; \boldsymbol{\Gamma}_{0}\right)
\end{array}\right),
$$

where $V_{12}$ and $V_{22}$ denote the potentials in the $\tilde{L}_{12}$ and $\tilde{L}_{22}$ components of $\tilde{L}$. In $\Delta L_{s}$ we expand the potentials in $\boldsymbol{\Gamma}-\boldsymbol{\Gamma}_{0}$,

$$
\begin{aligned}
V_{0}(\cdot ; \boldsymbol{\Gamma})-V_{0}\left(\cdot ; \boldsymbol{\Gamma}_{0}\right) & =\phi_{1}^{\prime}\left(\cdot, \boldsymbol{\Gamma}_{0}\right)\left(\Gamma_{1}-\Gamma_{0,1}\right)+\phi_{2}^{\prime}\left(\cdot, \boldsymbol{\Gamma}_{0}\right)\left(\Gamma_{2}-\Gamma_{0,2}\right)+\mathcal{O}\left(\left(T_{1}^{2}+\varepsilon^{2} T_{1}\right) V_{0}\right), \\
V_{i j}(\cdot ; \boldsymbol{\Gamma})-V_{i j}\left(\cdot, \boldsymbol{\Gamma}_{0}\right) & =\mathcal{O}\left(T_{1} V_{0}\right),
\end{aligned}
$$

where the $\mathcal{O}$ notation is in the sense of operators in the $X$ norm. The $\varepsilon^{2} T_{1}$ term in (4.34) arises from the amplitude variations of $V_{0}$ with $\boldsymbol{\Gamma}$. Using these expansions we see that

$$
\Delta L_{s} W=\left(\begin{array}{c}
2 \varepsilon^{-2}\left[\left(\Gamma_{1}-\Gamma_{0,1}\right) \phi_{1}^{\prime}+\left(\Gamma_{2}-\Gamma_{0,2}\right) \phi_{2}^{\prime}\right] W_{2}+\mathcal{O}\left(\left(\varepsilon^{-2} T_{1}^{2}+T_{1}\right) V_{0} W_{2}\right) \\
\mathcal{O}\left(T_{1} V_{0}\left(W_{1}+W_{2}\right)\right)
\end{array}\right)
$$

Moreover, the mass of $\phi_{k}^{\prime} W_{2}$ is $\mathcal{O}\left(\varepsilon^{4}\|W\|_{X}\right)$ since $W \in X_{\boldsymbol{\Gamma}_{0}}$ and hence $W \perp \Psi_{ \pm}^{\dagger}$. The mass of the first component of $\Delta L_{s} W$ arises from the higher order terms, and satisfies

$$
\left|\overline{\left[\Delta L_{s} W\right]_{1}}\right| \leq c\left(\varepsilon^{-2} T_{1}^{2}+T_{1}\right)\|W\|_{X}
$$

On the other hand the weighted norm of the first component satisfies

$$
\begin{aligned}
\left\|<x>\left[\Delta L_{s} W\right]_{1}\right\|_{L^{1}} & \leq c\left(\varepsilon^{-2} T_{1}\left\|<x>\phi_{j}^{\prime} W_{2}\right\|_{L^{1}}+\left(\varepsilon^{-2} T_{1}^{2}+T_{1}\right)\left\|<x>V_{0} W_{2}\right\|_{L^{1}}\right), \\
& \leq c\left(\varepsilon^{-2} T_{1}\left\|<x>\phi^{\prime}\right\|_{L^{1}}+\left(\varepsilon^{-2} T_{1}^{2}+T_{1}\right)\left\|<x>V_{0}\right\|_{L^{1}}\right)\|W\|_{X}, \\
& \leq c\left(\varepsilon^{-2}+\Delta \Gamma\right) T_{1}\|W\|_{X},
\end{aligned}
$$

where $\Delta \Gamma=\left|\Gamma_{1}-\Gamma_{2}\right|$ is the pulse separation. Combining the semi-group estimates (3.68) with (4.37-4.38) we obtain

$$
\left\|S(t-s) \tilde{\pi}_{0}\left(\Delta L_{s} W(s)\right)\right\|_{X} \leq M e^{-\nu(t-s)}\left(1+\varepsilon^{2} \Delta \Gamma\right) T_{1}(s)\|W\|_{X},
$$

where the $T_{1}^{2}\|W\|_{X}$ and $\varepsilon^{2} T_{1}\|W\|_{X}$ terms are dominated by $T_{1}\|W\|_{X}$ from the second component. Thus the dominant contribution from the secular terms comes from the second component of (4.36), the extra powers of $\varepsilon$ obtained by the approximate orthogonality of $W_{2}$ and $V_{0}^{\prime}$ are superfluous. So long as the pulses separation $\Delta \Gamma=\mathcal{O}\left(\varepsilon^{-2}\right)$, which is consistent with $\Gamma \in \mathcal{K}$, we may absorb the second term in (4.39) into the first.

While the small mass version of the semi-group estimates was not helpful for the secular term, it plays a key role on the impact of the reductive term, $\Delta L_{r} W$. Including the impact of the modification of the leading order potential, and neglecting the higher order terms in the potential, the reductive term is given by

$$
\Delta L_{r}=\left(\begin{array}{cc}
0 & 2 \varepsilon^{-2}\left(V_{0}-\delta_{\Gamma_{1}} \otimes \phi_{1}\left(\boldsymbol{\Gamma}_{0}\right)-\delta_{\Gamma_{2}} \otimes \phi_{2}\left(\boldsymbol{\Gamma}_{0}\right)\right)+\mathcal{O}\left(V_{0}\right) \\
\mathcal{O}\left(\varepsilon^{2} V_{0}^{2}\right) & \mathcal{O}\left(\varepsilon^{2} V_{0}\right)
\end{array}\right)
$$

where here the $\mathcal{O}$ indicates point-wise estimates. In fact the motivation behind the renormalization of the operator $L_{\boldsymbol{\Gamma}_{0}}$ to $\tilde{L}_{\boldsymbol{\Gamma}_{0}}$ is the observation that $\left(V_{0}-\delta_{\boldsymbol{\Gamma}_{1}} \otimes \phi_{1}-\delta_{\boldsymbol{\Gamma}_{2}} \otimes \phi_{2}\right) W$ has zero total mass and a smooth 
Fourier transform for any $W \in L^{2}$. Using this fact, the zero-mass version of the semi-group estimate (3.68), and the semi-strong assumption, $\Delta \Gamma=\mathcal{O}\left(\varepsilon^{-2}\right)$, it is straightforward to obtain the estimate

$$
\left\|S(t-s) \tilde{\pi}_{0}\left(\Delta L_{r} W(s)\right)\right\|_{X} \leq M e^{-\nu(t-s)}\left(\varepsilon^{2}+\varepsilon^{4} \Delta \Gamma\right)\|W\|_{X} .
$$

Finally for the nonlinear term, given by (3.5), it is easy to verify,

$$
\begin{aligned}
\left\|S(t-s) \tilde{\pi}_{0} \mathcal{N}\right\|_{X} & \leq M e^{-\nu(t-s)}\left(\left\|W_{2}^{2}\right\|_{L^{1}}+\left\|W_{2}^{2}\right\|_{L^{2}}+\left\|W_{1}^{2}\right\|_{L^{2}}\right), \\
& \leq M e^{-\nu(t-s)}\|W\|_{X}^{2} .
\end{aligned}
$$

From the bounds on the reduced residual (4.19-4.20) and the semi-group estimate we obtain

$$
\|S(t-s) \tilde{R}\|_{X} \leq M \varepsilon^{3} e^{-\nu(t-s)} .
$$

Taking the $X$-norm of variation of constants solution for $W,(4.25)$, and using the estimates outlined above we obtain

$$
\|W(t)\|_{X} \leq M\left(e^{-\nu \Delta t}\left\|W\left(t_{0}\right)\right\|_{X}+\int_{t_{0}}^{t} e^{-\nu(t-s)}\left[\varepsilon^{3}+\left(\varepsilon^{2}+T_{1}(s)\right)\|W(s)\|_{X}+\|W(s)\|_{X}^{2}\right] d s\right) .
$$

To estimate the decay of $\left\|W\left(t^{\prime}\right)\right\|_{X}$ for $t^{\prime} \in\left(t_{0}, t\right)$ we evaluate (4.45) at $t=t^{\prime}$, multiply by $e^{\nu\left(t^{\prime}-t_{0}\right)}$, and take the sup over $t^{\prime} \in\left(t_{0}, t\right)$ obtaining

$$
\begin{aligned}
T_{0}(t) & \leq M\left(T\left(t_{0}\right)+\int_{t_{0}}^{t}\left[\varepsilon^{3} e^{\nu\left(s-t_{0}\right)}+\left(\varepsilon^{2}+T_{1}(t)\right) T_{0}(t)+e^{-\nu s} T_{0}(t)^{2}\right] d s\right), \\
& \leq M\left(T_{0}\left(t_{0}\right)+\varepsilon^{3} e^{\nu \Delta t}+\left(\varepsilon^{2}+T_{1}(t)\right) \Delta t T_{0}(t)+T_{0}(t)^{2}\right) .
\end{aligned}
$$

From (4.32) we may eliminate $T_{1}$ from the $T_{0}$ estimate,

$$
T_{0}(t) \leq M\left(T_{0}\left(t_{0}\right)+\varepsilon^{3} e^{\nu \Delta t}+\varepsilon^{2}\left(\Delta t+(\Delta t)^{2}\right) T_{0}(t)+T_{0}(t)^{2}+\Delta t T_{0}^{3}\right)
$$

For $\Delta t \ll \min \left\{\varepsilon^{-1}, T_{0}^{-1}\right\}$ the term $M \varepsilon^{2}\left((\Delta t)^{2}+\Delta t\right)<\frac{1}{2}$ and we may eliminate the linear term in $T_{0}$ from the right-hand side, in addition we may absorb the cubic term in $T_{0}$ into the quadratic one. With these reductions (4.48) becomes

$$
T_{0} \leq 2 M\left(T_{0}\left(t_{0}\right)+\varepsilon^{3} e^{\nu \Delta t}+T_{0}^{2}\right)
$$

The quadratic equation in $T_{0}$

$$
0=T_{0}\left(t_{0}\right)+\varepsilon^{3} e^{\nu \Delta t}-\frac{1}{2 M} T_{0}+T_{0}^{2},
$$

has two positive real roots so long as $T_{0}\left(t_{0}\right)+\varepsilon^{3} e^{\nu \Delta t} \ll 1$. The smaller of these roots, $r_{0}$ takes the form

$$
r_{0}=2 M\left(T_{0}\left(t_{0}\right)+\varepsilon^{3} e^{\nu \Delta t}\right)+\mathcal{O}\left(T_{0}\left(t_{0}\right)+\varepsilon^{3} e^{\nu \Delta t}\right)^{2},
$$

while the larger is

$$
r_{1}=\frac{1}{2 M}+\mathcal{O}\left(T_{0}\left(t_{0}\right)+\varepsilon^{3} e^{\nu \Delta t}\right)
$$


Thus if $T_{0}\left(t_{0}\right) \ll 1$ and $\varepsilon^{3} e^{\nu \Delta t} \ll 1$ then there is an excluded region, either $0<T_{0}<r_{0}$ or $r_{1}<T_{0}<\infty$. Since $T_{0}\left(t_{0}\right)<r_{0}$ and $T_{0}$ is continuous in $t$, we see that

$$
T_{0}(t) \leq r_{0} \leq M\left(T_{0}\left(t_{0}\right)+\varepsilon^{3} e^{\nu \Delta t}\right)
$$

so long as

$$
\Delta t \leq \frac{3 \beta|\log \varepsilon|}{\nu}
$$

for any fixed $\beta<1$. This condition on $\Delta t$ prevents the secularity from dominating the linear operator, in particular it is a stronger condition on $\Delta t$ than that imposed after equation (4.48). This implies that

$$
\|W(t)\|_{X} \leq M\left(e^{-\nu\left(t-t_{0}\right)}\left\|W\left(t_{0}\right)\right\|_{X}+\varepsilon^{3}\right), \quad \text { for } t \in\left(t_{0}, t_{0}+\frac{3 \beta|\log \varepsilon|}{\nu}\right)
$$

and in particular for $t_{1}=t_{0}+\Delta t$ we have

$$
\left\|W\left(t_{1}\right)\right\|_{X} \leq M\left(\varepsilon^{3 \beta}\left\|W\left(t_{0}\right)\right\|_{X}+\varepsilon^{3}\right) .
$$

4.3. The RG Iteration. We break the time evolution into a series of inital value problems, tracking the decay of the remainder over the long-time scale of many RG iterations. We fix $\beta<1$ and $\Delta t=\frac{3 \beta|\log \varepsilon|}{\nu}$. The renormalization times are defined sequentially

$$
t_{n}=t_{n-1}+\Delta t
$$

We break the evolution of $W$ into disjoint intervals $I_{n}=\left[t_{n}, t_{n+1}\right)$. On each interval $I_{n}$ we solve the initial value problem (4.21) with initial data $W\left(t_{n}\right) \in X_{\boldsymbol{\Gamma}_{n}}$, with the quantities $T_{0, n}$ and $T_{1, n}$ corresponding to (4.23-4.24) over $I_{n}$. The renormalization map takes the initial data, $W_{(n-1)}=W\left(t_{n-1}\right)$ for the initial value problem on interval $I_{n-1}$ and returns the initial data $W_{n}=W\left(t_{n}\right)$ for the initial value problem on the interval $I_{n}$,

$$
\mathcal{G} W_{n-1}=W_{n} .
$$

Arguing inductively, the initial data and the new base point $\boldsymbol{\Gamma}_{n}$ are obtained from $W\left(t_{n}^{-}\right)$, the end-value of the evolution of $W$ over $I_{n-1}$, by applying Proposition 4.1. Indeed we know that $W\left(t_{n}^{-}\right) \in X_{\Gamma_{n-1}}$ and so from (4.3) we have

$$
\left|\boldsymbol{\Gamma}_{n}-\boldsymbol{\Gamma}\left(t_{n}^{-}\right)\right| \leq M_{0}\left\|W\left(t_{n}^{-}\right)\right\|_{X}\left|\boldsymbol{\Gamma}\left(t_{n}^{-}\right)-\boldsymbol{\Gamma}\left(t_{n-1}\right)\right| \leq M_{0}\left\|W\left(t_{n}^{-}\right)\right\|_{X} T_{1, n-1}(t) .
$$

From the estimates on $\Delta t$, and $T_{1, n-1}$, we bound the jump in $\boldsymbol{\Gamma}$ at renormalization by

$$
\left|\boldsymbol{\Gamma}_{n}-\boldsymbol{\Gamma}\left(t_{n}^{-}\right)\right| \leq M_{0}\left(|\log \varepsilon| \varepsilon^{2}+T_{0, n-1}^{2}\right)\left\|W\left(t_{n}^{-}\right)\right\|_{X} .
$$

The solution at time $t=t_{n}$ is independent of the decomposition,

$$
\vec{U}\left(t_{n}\right)=\Phi_{\boldsymbol{\Gamma}\left(t_{n}^{-}\right)}+W\left(t_{n}^{-}\right)=\Phi_{\boldsymbol{\Gamma}_{n}}+W_{n},
$$

and we may bound the jump in $W$ at each renormalization

$$
\left\|W\left(t_{n}^{-}\right)-W\left(t_{n}\right)\right\|_{X}=\left\|\Phi_{\boldsymbol{\Gamma}\left(t_{n}^{-}\right)}-\Phi_{\boldsymbol{\Gamma}_{n}}\right\|_{X} \leq c\left|\boldsymbol{\Gamma}_{n}-\boldsymbol{\Gamma}\left(t_{n}^{-}\right)\right| \leq M_{0}\left(|\log \varepsilon| \varepsilon^{2}+T_{0, n-1}^{2}\right)\left\|W\left(t_{n}^{-}\right)\right\|_{X},
$$

where we used the fact that $U_{0}$ is $\mathcal{O}(1) X$-Lipschitz in $\boldsymbol{\Gamma}$, as follows from (3.6) and (1.2). From (4.53), using the equality $T_{0, n-1}\left(t_{n-1}\right)=\left\|W_{n-1}\right\|_{X}$, we have the estimate

$$
T_{0, n-1} \leq M_{1}\left(\left\|W_{n-1}\right\|_{X}+\varepsilon^{3(1-\beta)}\right) .
$$


Combining the estimates (4.62) and (4.63) with (4.56), we obtain a bound on $\mathcal{G} W_{n-1}=W_{n}$,

$$
\left\|\mathcal{G} W_{n-1}\right\|_{X} \leq\left(1+M_{0}\left[|\log \varepsilon| \varepsilon^{2}+M_{1}^{2}\left(\left\|W\left(t_{n-1}\right)\right\|_{X}+\varepsilon^{3(1-\beta)}\right)^{2}\right]\right) M\left(\varepsilon^{3 \beta}\left\|W\left(t_{n-1}\right)\right\|_{X}+\varepsilon^{3}\right)
$$

Neglecting the terms involving positive powers of $\varepsilon$ within the first parenthesis on the left-hand side, we may bound $\left\|W\left(t_{n}\right)\right\|_{X}$ by $\eta_{n}$, solution of the map

$$
\eta_{n}=M\left(1+M_{2} \eta^{2}\right)\left(\varepsilon^{3 \beta} \eta_{n-1}+\varepsilon^{3}\right),
$$

with $\eta_{0}=\left\|W\left(\cdot, t_{0}\right)\right\|_{X}$, and $M_{2}=M_{0} M_{1}^{2}$. It is easy to see for $\eta_{0}=\mathcal{O}(1)$ and $\varepsilon$ sufficiently small that

$$
\eta_{n} \rightarrow \frac{M}{1-\varepsilon^{3 \beta} M} \varepsilon^{3}
$$

as $n \rightarrow \infty$. Since $\left\|W\left(\cdot, t_{n}\right)\right\|_{X} \leq \eta_{n}$, the estimate (4.56) yields the result (1.4) in Theorem 1.1.

4.4. Long-time Asymptotics. To recover the asymptotic pulse motion, we consider the situation where $t$ is sufficiently large that $\|W\|_{X} \leq M \varepsilon^{3}$. In this regime we see from (4.32) that $T_{1} \leq c \varepsilon^{2}|\log \varepsilon|$, and hence from (4.27) that

$$
\|\Delta L W\|_{L^{2}} \leq c \varepsilon^{2}|\log \varepsilon|\|W\|_{L^{2}} \leq c \varepsilon^{5}|\log \varepsilon|
$$

Moreover from the form (3.5) of the nonlinearity we readily verify that

$$
\left\|\mathcal{N}_{2}(W)\right\| \leq c\|W\|_{X}^{2}=\mathcal{O}\left(\varepsilon^{6}\right) .
$$

In this regime the estimates (4.67) and (4.68) on the secularity and the nonlinearity show that the remainder $W$ has an asymptotically small influence on the pulse evolution equations (4.17) which reduce to

$$
\dot{\Gamma}_{k}(t)=-\frac{\left(R_{2}, \phi_{k}^{\prime}(\cdot ; \boldsymbol{\Gamma}(t))\right)_{L^{2}}}{\left\|\phi_{k}^{\prime}\right\|_{L^{2}}^{2}}+\mathcal{O}\left(|\log \varepsilon| \varepsilon^{5}\right)
$$

Furthermore, the asymptotic form (2.16) for the second component of the remainder shows that

$$
\dot{\Gamma}_{k}(t)=\varepsilon^{2} \hat{c}(\boldsymbol{\Gamma}) \frac{\left(\phi_{1}^{\prime}-\phi_{2}^{\prime}, \phi_{k}^{\prime}\right)_{L^{2}}}{\left\|\phi_{k}^{\prime}\right\|_{L^{2}}^{2}}+\mathcal{O}\left(\varepsilon^{4}\right)=(-1)^{k+1} \varepsilon^{2} \hat{c}(\boldsymbol{\Gamma})+\mathcal{O}\left(\varepsilon^{4}\right),
$$

where $\hat{c}(\boldsymbol{\Gamma})$ is - by construction - the position dependent formal pulse speed given by (2.7). In particular the pulse separation $\Delta \Gamma=\Gamma_{1}-\Gamma_{2}$ grows as given by (1.5) while the amplitudes increases according to (2.10).

\section{Acknowledgments}

KP was supported by NSF grants DMS 04055965 and DMS 0510002. AD acknowledges support from NWO.

REFERENCES

[1] A. Doelman, W. Eckhaus and T. J. Kaper [2000], Slowly-modulated two-pulse solutions in the Gray-Scott model I: asymptotic construction and stability, SIAM J. Appl. Math. 61(3) 1080-1102. 
[2] A. Doelman, W. Eckhaus and T. J. Kaper [2001], Slowly-modulated two-pulse solutions in the Gray-Scott model II: geometric theory, bifurcations and splitting dynamics, SIAM J. Appl. Math. 61(6) 2036-2062.

[3] A. Doelman, S. Ei, T. Kaper, and K. Promislow, The nonlinear stability of semi-strongly interacting two-pulse solutions in the weakly damped Gray-Scott equations, in preparation.

[4] A. Doelman, R. A. Gardner, and T.J. Kaper [2001], Large stable pulse solutions in reaction-diffusion equations, Ind. Univ. Math. J., 50, 443-507.

[5] A. Doelman and T.J. Kaper [2002], Semi-strong pulse interactions in a class of reaction-diffusion equations, SIAM J. Appl. Dyn. Sys., 2, 53-96.

[6] A. Doelman and H. van der Ploeg [2001], Homoclinic stripe patterns, SIAM J. Appl. Dyn. Syst., 1(1), 65 - 104.

[7] S. Ei [2002], The motion of weakly-interacting pulses in reaction-diffusion systems, J. Dyn. Diff. Eq. 14, 85-137.

[8] S. Ei, M. Mimura, and M. Nagayama [2002] Pulse-pulse interactions in reaction-diffusion systems, Physica D 165, 176-198.

[9] A. Gierer and H. Meinhardt [1972], A theory of biological pattern formation, Kybernetik, 12, 30-39.

[10] R. Moore and K. Promislow [2005], Renormalization group reduction of pulse dynamics in thermally loaded optical parametric oscillators, Physica D 206 (2005) 62-81.

[11] Y. Nishiura and D. Ueyama [2001], Spatio-temporal chaos for the Gray-Scott model, Physica D 150, $137-162$.

[12] K. Promislow [2002], A renormalization method for modulational stability of quasi-steady patterns in dispersive systems, SIAM J. Math. Analysis 33 No. 6, 1455-1482.

[13] B. Sandstede [2002], Stability of travelling waves, pp. 983-1055 in 'Handbook of Dynamical Systems, II', B. Fiedler (ed.) [2002], Elsevier.

[14] M.J. Ward, W. Sun, and R. Russell [2005], The slow dynamics of two-spike solutions for the Gray-Scott and GiererMeinhardt systems: competition and oscillatory instabilities, to appear SIAM J. Appl. Dyn. Sys. 